\title{
Two-shell vortex and antivortex dynamics in a Corbino superconducting disk
}

\author{
L. R. E. Cabral, ${ }^{1,}{ }^{*}$ Belisa R. C. H. T. de Aquino, ${ }^{1,2}$ Clécio C. de Souza Silva, ${ }^{1}$ Milorad V. Milošević, ${ }^{2}$ and François M. Peeters ${ }^{2}$ \\ ${ }^{1}$ Departamento de Física, Universidade Federal de Pernambuco, Recife 50670-901, Brazil \\ ${ }^{2}$ Departement Fysica, Universiteit Antwerpen, Groenenborgerlaan 171, B-2020 Antwerpen, Belgium
}

(Received 6 November 2015; published 20 January 2016)

\begin{abstract}
We examine theoretically the dynamics of two vortex shells in pinning-free superconducting thin disks in the Corbino geometry. In the first considered case, the inner shell is composed of vortices and the outer one of antivortices, corresponding to a state induced by the stray field of an off-plane magnetic dipole placed on top of the superconductor. In the second considered case, both shells comprise vortices induced by a homogeneous external field. We derive the equation of motion for each shell within the Bardeen-Stephen model and study the dynamics analytically by assuming both shells are rigid and commensurate. In both cases, two distinct regimes for vortex shell motion are identified: For low applied currents the entire configuration rotates rigidly, while above a threshold current the shells decouple from each other and rotate at different angular velocities. Analytical expressions for the decoupling current, the recombination time in the decoupled phases, as well as the voltage-current characteristics are presented. Our analytical results are in excellent agreement with numerical molecular dynamics simulations of the full many-vortex problem.
\end{abstract}

DOI: 10.1103/PhysRevB.93.014515

\section{INTRODUCTION}

The nonequilibrium dynamics of vortex matter in superconductors have attracted much attention in the past few decades [1-9]. Besides its crucial role in understanding the transport properties of superconducting materials, moving vortex matter exhibits a variety of properties relevant for problems as diverse as microscopic friction [10-12] and the nature of dynamical phase transitions $[6,13,14]$. A salient advantage of adopting vortex matter as a model system to investigate these phenomena lies in the fact that their dynamical properties are experimentally accessible over a wide range of control parameters, such as temperature, concentration, and shear drive.

One of the main experimental difficulties for studying vortex dynamics is the "contamination" of the dynamical phase under investigation caused by vortices which enter or exit through the sample edges. This can be avoided by performing experiments in the so-called Corbino-disk geometry, where current flows radially from the center towards the edges of a superconducting disk [15-19]. This way, vortices move azimuthally, without ever leaving the sample. For instance, the transition between Bragg glass and disordered solid phases in $\mathrm{NbSe}_{2}$ crystals is better depicted in experiments performed in the Corbino geometry than those in strips $[15,17]$. In addition, the Corbino geometry has been used to probe the vortex lattice melting transition in $\mathrm{Bi}_{2} \mathrm{Sr}_{2} \mathrm{CaCu}_{2} \mathrm{O}_{8+\delta}$ single crystals $[16,18]$ and to study metastable resistive states of thick $\mathrm{Mo}_{x} \mathrm{Si}_{1-x}$ films near the critical temperature [20].

In the Corbino geometry novel dynamical phases can appear induced by the strong inhomogeneity of the current density (which changes with radial distance as $1 / r$ ) [21-29]. The transition between such phases has been identified experimentally in weak pinning materials [24,28], as well as investigated both theoretically [23,27], in the context of the continuum approximation, and numerically [26,29] in

*1recabral@df.ufpe.br large vortex systems. First, at least two phases are noticeable: a rigid-body $(\mathrm{RB})$ rotation phase for small applied currents and a phase characterized by plastic motion of vortices for high currents, separated by a critical current $I_{\mathrm{dc}}[23-27,30]$. In the former phase, the vortex-vortex interaction is strong enough to form a firm vortex lattice rotating as a RB. The vortex velocities increase linearly with distance from the center of the disk in this case. In the latter, the shear stress generated by the inhomogeneous current density tears the $\mathrm{RB}$ motion into vortex rings rotating slower with increasing distance from the center of the disk [23,24,27]. This plastic motion evolves to laminar flow for further increased applied currents [26,29]. This state is characterized by the presence of topological defects in the vortex lattice which extend radially inside the disk [25,26]. Moreover, the unbinding of adjacent layers has also been observed in other systems of particles such as colloids in rotational drive [31], as well as in other geometries of the applied shear stress [32-35].

Theoretical [36-38] and experimental [39,40] work on disks containing a small number of vortices confirm that these systems naturally stabilize static configurations comprising vortex shells. Further theoretical investigations on the dynamical response of these systems within the Corbino setup suggest that the transition between RB motion and decoupled vortex-ring rotation depends on the particular vortex configuration, as well as on the external magnetic field [41-43]. Several interesting phenomena were observed, such as a nonmonotonic dependence of the critical current $I_{\mathrm{dc}}$ on the magnetic field, current-induced structural transitions between two stable vortex configurations, and multistep decoupling of vortex rings. In these works, the temperature-induced transition between the RB and the decoupled moving phases (referred to as the angular melting transition) has also been addressed. It has been suggested that the angular melting takes place at lower temperatures than the so-called radial melting, where the vortex shell structure disappears, and that this could explain the experimental results observed in untwinned YBCO single crystals in the Corbino geometry [24]. For larger vortex systems in mesoscopic disks it was even 
found that one vortex shell rotates decoupled from its adjacent vortex shells, while these shells remain locked in RB motion [43]. This reveals the importance of the (in)commensurability between adjacent shells for the dynamic phases appearing in the Corbino geometry.

Despite all these advances in understanding dynamical phases and phase transitions in the Corbino geometry, analytic considerations are still scarce. In the present work we aim at filling this gap by considering theoretically the cases where vortices arrange into two shells only. Such systems allow for an analytical approach and can be understood as the minimal systems to study the RB and decoupled phases as well as the transition between them and thereby shed light on further possibilities in more complex configurations.

Our model assumes two commensurate vortex shells and ignores the intrashell dynamics; that is, each shell is assumed to move rigidly. We consider not only the cases where both shells are composed of vortices but also configurations with vortex and antivortex shells, such as those found in hybrid superconductor-ferromagnetic systems [44-51]. In Ref. [52], we presented preliminary results of molecular dynamics simulation of the latter case. Here we develop a fully analytical approach for the case of two vortex shells with the same number of (anti-)vortices, which well describes both dynamical phases and establishes the conditions for observing either of them. For the more general case of commensurate vortex shells of either similar or dissimilar vorticities, we use an empirical ansatz which allows us to find analytic expressions for the recombination frequency and for the voltage-current characteristics of the system. The analytical results are then corroborated by the results of the numerical simulations.

This paper is organized as follows. In Sec. II we present the theoretical formalism used to develop our analytical model and to perform the molecular dynamics simulations. The analytical results for the intershell motion for equal number of vortices in each shell are presented in Sec. III. In Sec. IV, we extend the model to consider shells with different, but commensurate, numbers of vortices. The expressions for the voltage-current characteristics are derived in Sec. V. Finally, in Sec. VI, we show results of numerical simulations for both the vortexantivortex shells under an off-plane magnetic dipole and the vortex shells in homogenous magnetic field and compare them to our analytical results. The conclusions are drawn in Sec. VII.

\section{THEORETICAL APPROACH}

We consider a thin superconducting disk of thickness $d \leqslant \xi$ and radius $R=100 \xi$ in the limit of negligible demagnetization effects, i.e., for effective penetration depth $\Lambda=\lambda^{2} / d \gg R$. We consider two scenarios: (i) a magnetic dipole with magnetic moment $\mathbf{m}=m_{0} \hat{z}$ is placed along the disk symmetry axis (the $z$ axis) at a distance $z_{0}$ above the disk [cf. Fig. 1(a)] or (ii) a homogeneous magnetic field, $\mathbf{H}_{\mathbf{a}}$, is applied along the disk symmetry axis. In both cases, an external electric current $I$ is injected in the center and collected at the edge of the disk.

The vortex interactions are calculated analytically within the London approach [36,38] for vanishing and nonoverlapping vortex cores, in the London gauge, $\nabla \cdot \mathbf{A}=0$, and
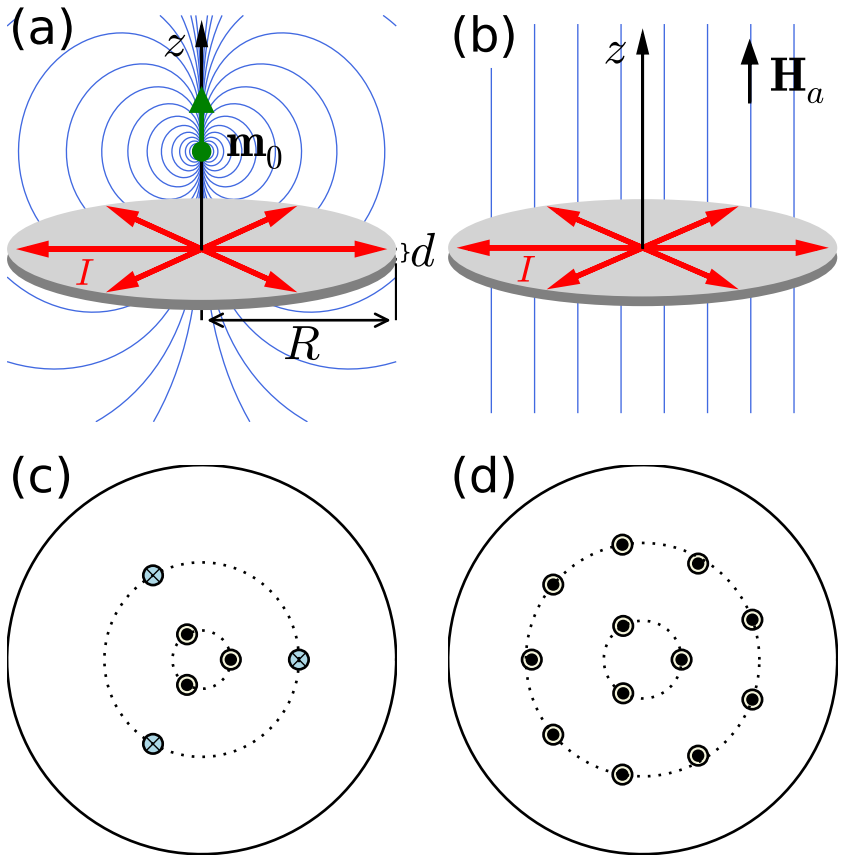

FIG. 1. Schematic representation of the studied system. (a) A magnetic dipole is placed at a distance $z_{0}$ from the superconducting disk of thickness $d$ and radius $R$. (b) Instead of a magnetic dipole, a homogeneous magnetic field $H_{a}$ is applied along $z$. For the Corbino disk an external current, $I$, is injected in the center of the disk and collected at the disk edge. Configurations containing (c) vortices (circles with dots) and antivortices (circles with crosses), as well as (d) with only vortices, are schematically shown for the cases depicted in (a) and (b), respectively.

disregarding demagnetization effects, obeying the equation

$$
\mu_{0} \Lambda \mathbf{J}+\mathbf{A}_{\mathrm{ext}}=\sum_{i=1}^{N_{c}} v_{i} \Phi\left(\mathbf{r}, \mathbf{r}_{i}\right) .
$$

where $\Phi_{0}=h / 2 e$ is the flux quantum, $\mathbf{r}_{i}$ is the position of the $i$ th vortex core, $N_{c}$ is the number of vortex cores, and $v_{i}$ is the number of fluxoids carried by the vortex $\left(v_{i}=-1\right.$ for antivortices and $\left|v_{i}\right|>1$ for giant vortices [53-55]). $\mathbf{A}_{\text {ext }}$ is the vector potential of the external magnetic fields, $\mathbf{J}(\mathbf{r}, 0)=\int_{0}^{d} d z \mathbf{j}(\mathbf{r}, z)$ is the sheet current density, and the function $\Phi\left(\mathbf{r}, \mathbf{r}_{i}\right)$ for a thin disk is given by

$$
\Phi\left(\mathbf{r}, \mathbf{r}_{i}\right)=\frac{v_{i} \Phi_{0}}{2 \pi} \hat{z} \times\left[\frac{\left(\mathbf{r}-\mathbf{r}_{i}\right)}{\left|\mathbf{r}-\mathbf{r}_{i}\right|^{2}}-\frac{\left(\mathbf{r}-\frac{R^{2}}{r_{i}^{2}} \mathbf{r}_{i}\right)}{\left|\mathbf{r}-\frac{R^{2}}{r_{i}^{2}} \mathbf{r}_{i}\right|^{2}}\right],
$$

where $\hat{z}$ is a unit vector directed along the $z$ axis. The forces acting on the $i$ th vortex are calculated using $\mathbf{F}_{i}=$ $v_{i} \Phi_{0} \mathbf{J}\left(\mathbf{r}_{i}\right) \times \hat{z}$, where $\mathbf{r}_{i}$ is the vortex position and $\mathbf{J}\left(\mathbf{r}_{i}\right)$ is the sheet current. Note again that the applied current $I$ gives rise to an inhomogeneous current density which decays as $1 / r$.

The vortex motion is described by Bardeen-Stephen equation [56]

$$
\mathbf{v}_{i}=\mathbf{F}_{i}^{I}+\mathbf{F}_{i}^{\mathrm{dip}}+\mathbf{F}_{i}^{a}+\mathbf{F}_{i}^{\text {self }}+\sum_{\substack{j=1 \\ i \neq j}}^{N_{c}} \mathbf{F}_{i j}
$$


with

$$
\begin{gathered}
\mathbf{F}_{i}^{I}=\frac{v_{i} I \mathbf{r}_{i} \times \hat{z}}{r_{i}^{2}}, \\
\mathbf{F}_{i}^{\mathrm{dip}}=-\frac{v_{i} m_{0} \mathbf{r}_{i}}{\left(r_{i}^{2}+z_{0}^{2}\right)^{3 / 2}}, \\
\mathbf{F}_{i}^{a}=-2 v_{i} H_{a} \mathbf{r}_{i}, \\
\mathbf{F}_{i}^{\text {self }}=\frac{2 v_{i}^{2} \mathbf{r}_{i}}{1-r_{i}^{2}}, \\
\mathbf{F}_{i j}=2 v_{i} v_{j}\left[\frac{r_{i}^{2}-\mathbf{r}_{j}-\mathbf{r}_{j}}{\left|\mathbf{r}_{i}-\mathbf{r}_{j}\right|^{2}}-\frac{r_{i}^{2} r_{j}^{2}-2 \mathbf{r}_{i} \cdot \mathbf{r}_{j}}{1+r_{i}}\right],
\end{gathered}
$$

where $\mathbf{F}_{i}^{I}$ represents the forces acting on vortex $i$ due to the applied current $I, \mathbf{F}_{i}^{\text {dip }}$ and $\mathbf{F}_{i}^{a}$ are the forces due to the shielding currents in response to the dipole field and/or the homogeneous magnetic field, $\mathbf{F}_{i}^{\text {self }}$ is the force produced by the vortex "own image," and $\mathbf{F}_{i, j}$ are the forces due to all other vortices. The length, time, magnetic moment of the magnetic dipole $m_{0}$, magnetic field, and electrical current $I$ are given in units of $R, \eta 4 \pi \mu_{0} \Lambda R^{2} / \Phi_{0}^{2}, \Phi_{0} R / \mu_{0}, \Phi_{0} / \mu_{0} \pi R^{2}$, and $\Phi_{0} / 2 \mu_{0} \Lambda$, respectively, where $\eta$ is the viscous drag coefficient appearing in the Bardeen-Stephen model. In order to avoid divergences, the ansatz $\left|\mathbf{r}_{i}-\mathbf{r}_{j}\right|^{2} \rightarrow \sqrt{\left|\mathbf{r}_{i}-\mathbf{r}_{j}\right|^{2}+2 \xi^{2}}$ was used in the simulations.

It is convenient to project Eqs. (3)-(8) along the $i$ thvortex radial and angular directions. For that, we take the vector and scalar products of $\mathbf{v}_{i}=\omega_{i} \times \mathbf{r}_{i}+v_{i, r} \frac{\mathbf{r}_{i}}{r_{i}}$ with $\mathbf{r}_{i}=$ $\left(r_{i} \cos \phi_{i}, r_{i} \sin \phi_{i}\right)$, which yields

$$
\begin{gathered}
\mathbf{r}_{i} \times \mathbf{v}_{i}=\mathbf{r}_{i} \times \mathbf{F}_{i}^{I}+\sum_{\substack{j=1 \\
i \neq j}}^{N_{c}} \mathbf{r}_{i} \times \mathbf{F}_{i j}, \\
\mathbf{r}_{i} \cdot \mathbf{v}_{i}=\mathbf{r}_{i} \cdot \mathbf{F}_{i}^{\mathrm{dip}}+\mathbf{r}_{i} \cdot \mathbf{F}_{i}^{a}+\mathbf{r}_{i} \cdot \mathbf{F}_{i}^{\mathrm{self}}+\sum_{\substack{j=1 \\
i \neq j}}^{N_{c}} \mathbf{r}_{i} \cdot \mathbf{F}_{i j},
\end{gathered}
$$

where $\mathbf{r}_{i} \times \mathbf{F}_{i}^{\text {dip }}=\mathbf{r}_{i} \times \mathbf{F}_{i}^{a}=\mathbf{r}_{i} \times \mathbf{F}_{i}^{\text {self }}=0$ and $\mathbf{r}_{i} \cdot \mathbf{F}_{i}^{I}=0$.

For low-enough applied current one expects the entire configuration to rotate as a whole. The angular velocity $\omega_{\mathrm{RB}}$ for this RB rotation can be derived from Eq. (9), since the sum of all the internal torques should be zero, i.e., $\sum_{i=1}^{N_{c}} \sum_{j=1}^{N_{c}} \mathbf{r}_{i} \times$ $\mathbf{F}_{i j}=0$. Therefore, by summing Eq. (9) for all the vortices and antivortices and noticing that $\omega_{i}=\omega_{\mathrm{RB}}$, for $i=1,2, \ldots, N_{c}$ in this case, we have $\omega_{\mathrm{RB}} \sum_{i=1}^{N_{c}} r_{i}^{2}=-I \sum_{i=1}^{N_{c}} \nu_{i}$. This yields

$$
\omega_{\mathrm{RB}}=-\frac{L I}{\sum_{i=1}^{N_{c}} r_{i}^{2}},
$$

where $L=\sum_{i=1}^{N_{c}} v_{i}$ is the total vorticity.

Using that $\mathbf{r}_{i} \times \mathbf{r}_{j}=-r_{i} r_{j} \sin \left(\phi_{i}-\phi_{j}\right) \hat{z}$ and $\mathbf{r}_{i} \cdot \mathbf{r}_{j}=$ $r_{i} r_{j} \cos \left(\phi_{i}-\phi_{j}\right)$, Eqs. (9) and (10) become

$$
r_{i}^{2} \omega_{i}=-v_{i} I+\sum_{\substack{j=1 \\ i \neq j}}^{N_{c}} \frac{2 v_{i} v_{j} r_{i} r_{j} \sin \phi_{i j}}{D_{1}\left(r_{i}, r_{j}, \phi_{i j}\right)}-\sum_{j=1}^{N_{c}} \frac{2 v_{i} v_{j} r_{i} r_{j} \sin \phi_{i j}}{D_{1}\left(1, r_{i} r_{j}, \phi_{i j}\right)},
$$

$$
\begin{aligned}
v_{i, r}= & -\frac{v_{i} m_{0} r_{i}}{\left(r_{i}^{2}+z_{0}^{2}\right)^{3 / 2}}-2 v_{i} H_{a} r_{i} \\
& +\sum_{\substack{j=1 \\
i \neq j}}^{N_{c}} \frac{2 v_{i} v_{j}\left(r_{i}-r_{j} \cos \phi_{i j}\right)}{D_{1}\left(r_{i}, r_{j}, \phi_{i j}\right)} \\
& -\sum_{j=1}^{N_{c}} \frac{2 v_{i} v_{j}\left(r_{j}^{2} r_{i}-r_{j} \cos \phi_{i j}\right)}{D_{1}\left(1, r_{i} r_{j}, \phi_{i j}\right)},
\end{aligned}
$$

where $\phi_{i j}=\phi_{i}-\phi_{j} \quad$ and $\quad D_{n}(x, y, \gamma)=x^{2 n}+y^{2 n}-$ $2 x^{n} y^{n} \cos (n \gamma)$. Notice that the terms inside the sums in Eq. (12) are the torques between the $j$ th and the $i$ th vortices, while in Eq. (13) they are the radial forces acting on the $i$ th vortex.

In the next sections, we apply this theory for the cases where the vortex configurations in the superconducting disk form two concentric shells with, eventually, a vortex or giant vortex near the center. As we discuss in Sec. VI, the contribution of such vortex to the shell dynamics is negligible. However, it does influence the RB rotation as it contributes to the total vorticity of the system [see Eq. (11)].

Our analytical results were derived in the limit where the ratio of the radial positions of the inner to the outer shell is much smaller than 1 (but expressions for any ratio value are obtained in Appendix D) and for an infinite superconducting film. However, they are very reasonable approximations for superconducting disks where the two shells are not too close to each other and both are far from the disk border, as supported by the numerical simulation results presented in Sec. VI. Our main results are summarized as follows.

(i) For the case with zero total vorticity Eqs. (25)-(29) describe the intershell motion. Equation (21) gives the decoupling current.

(ii) For commensurate vortex shells Eqs. (37)-(44) yield a description of the intershell motion. For the particular case of coexisting vortices and antivortices Eq. (47) and Eq. (48) give the threshold current where the antivortex shell reverses its motion.

(iii) Results for the $V-I$ characteristics are given by Eqs. (51)-(53) and Eqs. (56)-(58).

\section{INTERSHELL MOTION FOR ZERO TOTAL VORTICITY: ANALYTICAL RESULTS}

In order to better understand the intervortex shell dynamics in Corbino geometry, we considered configurations with two concentric shells in the superconducting disk containing the same number of vortices; i.e., each shell contains $N_{1}=N_{2}=$ $N$ vortices (or antivortices). All vortices in each shell have the same radial positions $R_{m}$, where $m=1,2$. The system has $N$-fold rotational symmetry, with the vortices placed at the vertices of coaxial regular polygons with $N$ sides. Each vortex is described by its position $\left(R_{m} \cos \phi_{m, i}, R_{m} \sin \phi_{m, i}\right)$ and vorticity $v_{m}=1 \quad\left(v_{m}=-1\right.$ for an antivortex $)$, where $\phi_{m, i}=\varphi_{m}+2 \pi i / N$, and $\varphi_{m}=\varphi_{+}$is an arbitrary angular position for the $m$ th shell. We further assume that the vortices within the vortex shell remain rotating with the same angular 
velocity. This means that, as long as that assumption holds, the interring torques within the same shell cancel out.

Under the above assumptions we find the following equations of motion for each vortex shell,

$$
\begin{gathered}
R_{m}^{2} \omega_{m}=-v_{m} I+\tau_{m n}, \\
v_{m, r}=-\frac{v_{m} m_{0} R_{m}}{\left(R_{m}^{2}+z_{0}^{2}\right)^{3 / 2}}+\frac{N-1}{R_{m}}+\frac{2 N_{-} R_{m}^{2 N-1}}{1-R_{m}^{2 N}}+f_{m n},
\end{gathered}
$$

where $\varphi_{m n}=\varphi_{m}-\varphi_{n}$, with either $m=1$ and $n=2$ or $m=2$ and $n=1$. The torques produced by the ring $n$ on a given vortex in the $m$ th ring $\tau_{m n}$ and the radial forces between the shells are given, respectively, by Eqs. (A3) and (A4).

In the rest of this section we focus on configurations with two shells and the same number of vortices and antivortices in both shells (although the above equations remain valid for two shells with the same number of vortices with $v=$ 1). In this case $L=0$, "+" and "-" are labels for the vortex and antivortex shells, respectively, and $N_{+}=N_{-}=N$. Rigorously speaking, the neutrality $(L=0)$ of the vortex system is guaranteed only for a disk with infinite radius, for which the total flux induced by the nearby magnetic dipole is zero [49]. Nevertheless, for finite but sufficiently large disks, $L=0$ configurations are still likely to occur $[48,50]$.

Figure 2 depicts a typical configuration with no vortex at the center, five vortices at the inner shell, and five antivortices at the outer shell, which we refer to as the $(0,5,-5)$ configuration. Notice that the symmetry of the model yields that all the (anti)vortices within the same shell move together. The equations for angular motion of the vortex $(+)$ and the antivortex $(-)$ shells become

$$
R_{ \pm}^{2} \omega_{ \pm}=\mp\left(I-\frac{\tau_{+-}}{N}\right)
$$

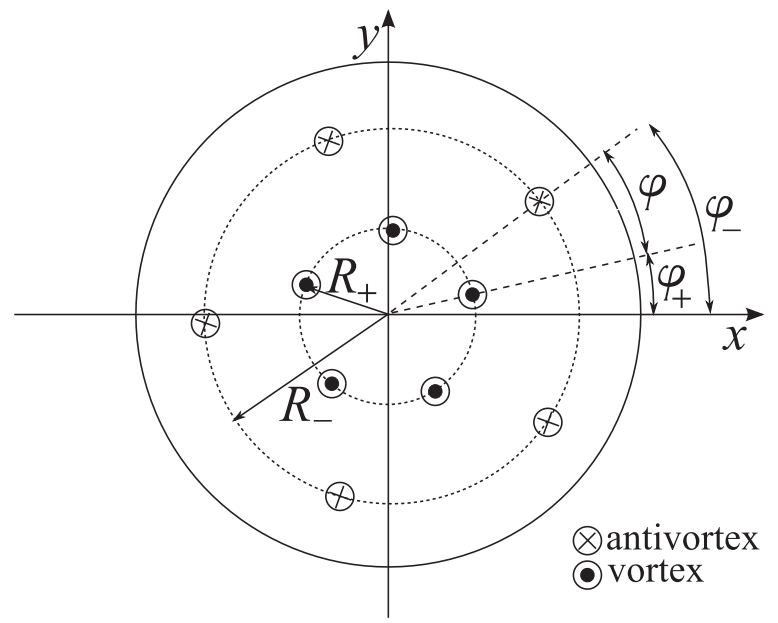

FIG. 2. The two-vortex-shell model for configurations with the same number of vortices and antivortices in each shell is represented schematically for the $(0,5,-5)$ configuration as an example. Circles with dots (crosses) represent vortices (antivortices). The vortices (antivortices) are at radius $R_{+}\left(R_{-}\right)$and at angular positions $\varphi_{+}+2 \pi j / N\left(\varphi_{-}+2 \pi j / N\right)$, for $j=0,1, \ldots, N . \varphi=\varphi_{-}-\varphi_{+}$is the intershell angular displacement. where $\tau_{-+}=-\tau_{+-}$are the intershell torques,

$$
\begin{aligned}
\frac{\tau_{+-}}{N}= & 2 N R_{-}^{N} R_{+}^{N}\left[\frac{1}{D_{N}\left(R_{+}, R_{-}, \varphi\right)}\right. \\
& \left.-\frac{1}{D_{N}\left(1, R_{+} R_{-}, \varphi\right)}\right] \sin N \varphi .
\end{aligned}
$$

From these equations we obtain the relative angular velocity between the antivortex and the vortex shells,

$$
\omega_{-}-\omega_{+}=\frac{d \varphi}{d t}=\left(\frac{1}{R_{+}^{2}}+\frac{1}{R_{-}^{2}}\right)\left(I-\frac{\tau_{+-}}{N}\right),
$$

where $\varphi=\varphi_{-}-\varphi_{+}$. It is reasonable to assume that the radial vortex positions do not change much for nonzero $I$. In this case, the radial positions obtained for the $I=0$ case (cf. Appendix A), i.e., $R_{+}=R_{+}(0)$ and $R_{-}=R_{-}(0)$, can be used in the equation describing the intershell motion.

We proceed by neglecting the contribution from the vortex images since it is much smaller than the nonimage term because $R_{+}<R_{-}<1$. In fact, we find $R_{-} \lesssim 0.5$ and $R_{+} \lesssim 0.2$ as typical values for the shells radii, which gives $D_{N}\left(1, R_{+} R_{-}, \varphi_{+-}\right)^{-1} \sim 1$, while $D_{N}\left(R_{+}, R_{-}, \varphi_{+-}\right)^{-1} \gg 1$. To give some examples, for $R_{-}=$ $0.5, R_{+}=0.2$ and $N=5$ we have $D_{N}\left(R_{+}, R_{-}, \varphi_{+-}\right)^{-1} \sim$ $10^{3}$ and $D_{N}\left(1, R_{+} R_{-}, \varphi_{+-}\right)^{-1} \sim 1$, while for for $R_{-}=$ $0.5, R_{+}=0.2$ and $N=2,10<D_{N}\left(R_{+}, R_{-}, \varphi_{+-}\right)^{-1}<25$ and $D_{N}\left(1, R_{+} R_{-}, \varphi_{+-}\right)^{-1} \sim 1$. Note that this approximation becomes exact for an infinite film. Therefore,

$$
\frac{\tau_{+-}}{N} \approx \frac{2 N \varrho^{N} \sin N \varphi}{1+\varrho^{2 N}-2 \varrho^{N} \cos N \varphi},
$$

where $\varrho=R_{+} / R_{-}$. Finally, the equation for intershell motion is obtained by substituting Eq. (19) in Eq. (18), which yields

$$
\frac{d \vartheta}{d t}=K\left[I-\frac{2 N \varrho^{N} \sin \vartheta}{1+\varrho^{2 N}-2 \varrho^{N} \cos \vartheta}\right],
$$

where $\vartheta=N \varphi$ and $K=N\left(R_{+}^{-2}+R_{-}^{-2}\right)$. This formula gives the intershell dynamics, since it shows that $\varphi$ grows while $I$ increases.

Inspection of Eq. (20) allows us to identify two distinct regimes of the intershell dynamics. For small-enough currents the shells are coupled to each other because of the attractive vortex-antivortex interaction. In this case, both shells are expected to rotate with $\mathrm{RB}$ angular velocity $\omega_{\mathrm{RB}}$, given by Eq. (11). However, since $L=0$, we have $\omega_{\mathrm{RB}}=0$. Therefore, the stationary regime for small currents corresponds to no rotation of either shell. This phase extends until a current strong enough to unbind the shells is reached. This decoupling current, $I_{\mathrm{dc}}$, can be calculated by maximizing the right-hand side (RHS) of Eq. (20), $I=\frac{2 N \varrho^{N} \sin \vartheta}{1+\varrho^{2 N}-2 \varrho^{N} \cos \vartheta}$, with respect to $\vartheta$. This yields

$$
I_{\mathrm{dc}}=\frac{2 N \varrho^{N}}{1-\varrho^{2 N}}
$$

and

$\vartheta_{c}=N \varphi_{c}=\arccos \left(\frac{2 \varrho^{N}}{1+\varrho^{2 N}}\right)=\arcsin \left(\frac{1-\varrho^{2 N}}{1+\varrho^{2 N}}\right)$, 
where $\varphi_{c}$ is the maximal angular displacement between the vortex and the antivortex shells with no intershell motion. For $I>I_{\mathrm{dc}}$ the stationary regime corresponds to $\varphi$ oscillating in time, with the vortex and antivortex shells moving at different angular velocities.

Full analytical solution of Eq. (20) is given in detail in Appendix D. The resulting expressions are not very illuminating. However, in most of the cases we find $R_{+} \ll R_{-}$, which allows Eqs. (D3), (D4), and (D5) to be significantly simplified, since the denominator of Eq. (20) is approximately equal to 1 . The intershell equation of motion becomes

$$
\frac{d \vartheta}{d t}=K\left(I-I_{\mathrm{dc}} \sin \vartheta\right)
$$

where $I_{\mathrm{dc}}=2 N\left(R_{+} / R_{-}\right)^{N}$. This equation has a solution monotonically increasing or decreasing with time (depending on the initial conditions) for $I \leqslant I_{\mathrm{dc}}$, while a periodic solution is expected for $I>I_{\mathrm{dc}}$. In fact, for $I<I_{\mathrm{dc}}$ the solution reaches a stationary value,

$$
\vartheta_{\text {stat }}=N \varphi_{\text {stat }}=\arcsin \left(\frac{I}{I_{\mathrm{dc}}}\right),
$$

which can be readily computed from the above equation by setting $\frac{d \vartheta}{d t}=0$.

Equation (23) is solved in Appendix B. After some algebraic manipulation we find

$$
\varphi=\frac{2}{N} \arctan [\zeta(t)]
$$

where

$$
\zeta(t)=\frac{\tan \left(\frac{N \varphi_{0}}{2}\right)+\left[\tilde{I}-\tan \left(\frac{N \varphi_{0}}{2}\right)\right] f(I, t)}{1+\left[1-\tilde{I} \tan \left(\frac{N \varphi_{0}}{2}\right)\right] f(I, t)},
$$

and

$$
f(I, t)= \begin{cases}\frac{\tanh (\pi t / T)}{\sqrt{1-\tilde{I}^{2}},} & I<I_{\mathrm{dc}}, \\ \frac{\tan (\pi t / T)}{\sqrt{\tilde{I}^{2}-1}}, & I>I_{\mathrm{dc}} .\end{cases}
$$

In the equations above, $\tilde{I}=I / I_{\mathrm{dc}}, \varphi_{0}=\varphi(t=0)$, and

$$
T=\frac{2 \pi}{K I_{\mathrm{dc}} \sqrt{\left|\tilde{I}^{2}-1\right|}}=\frac{\frac{2 \pi}{N}}{\left(\frac{1}{R_{+}^{2}}+\frac{1}{R_{-}^{2}}\right) \sqrt{\left|I^{2}-I_{\mathrm{dc}}^{2}\right|}} .
$$

For $I>I_{\mathrm{dc}}$ this equation gives the period of $\varphi$ (which is the time it takes for the vortex and antivortex shells to detach, slide past each other, and reconnect again). For $I<I_{\mathrm{dc}}$ it represents a characteristic time in which $\varphi$ reaches its stationary value [in Appendix C we show that Eq. (24) is the limit of Eqs. (25), (26), and (27) when $t \gg T$ for $\left.I<I_{\mathrm{dc}}\right]$.

To be specific, we consider the case of two shells with $N_{+}=N_{-}=4$ for $m_{0}=1.5$ and $z_{0}=0.045$. The equilibrium values of the shells radii were obtained analytically following the stabilization procedure described in Appendix A, which yields $R_{+}=0.014545$ and $R_{-}=0.289462$. The time dependence of $\varphi$ is depicted in Fig. 3(a) for $I<I_{\mathrm{dc}}$ and in Fig. 3(b) for $I>I_{\mathrm{dc}}$ [Eqs. (26) and (27)]. The behavior shown in Fig. 3(a) is related to the transient time, after the external current is switched on, the configuration [initially
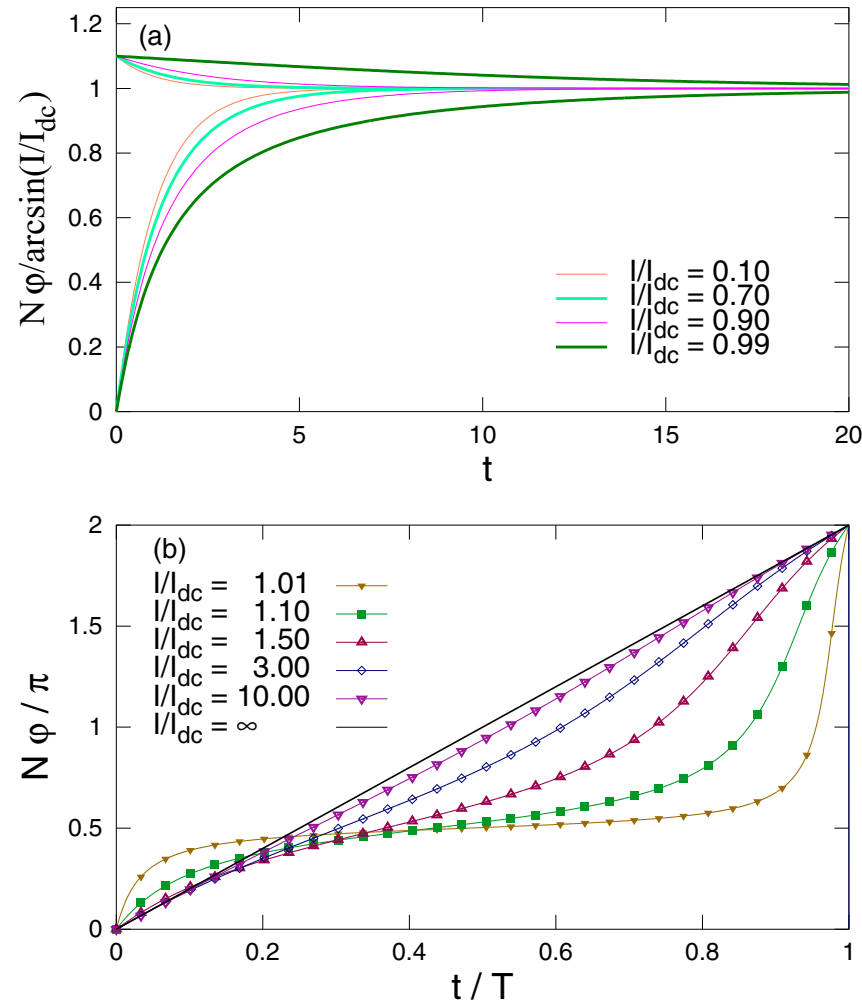

FIG. 3. The intershell angular displacement $\varphi$ as function of time for $R_{+}=0.014545$ and $R_{-}=0.289462$ (i.e., $R_{+} / R_{-} \leqslant 1$ ) for the $(0,4,-4)$ vortex configuration (obtained for $m_{0}=1.5$ and $\left.z_{0}=0.045\right)$. For $I<I_{\mathrm{dc}}$ (a) we show $\varphi(t)$ approaching the limiting value given by Eq. (22) for two sets of initial conditions: $\varphi(t=$ $0)=0$ and $\varphi(t=0)=1.1 \arcsin \left(I / I_{\mathrm{dc}}\right) / N$. For $I>I_{\mathrm{dc}}$ (b) $\varphi$ varies periodically. The time is given in units of the period $T$ of the vortex intershell decoupling and reconnection.

at $\varphi_{0}=0$ or $\left.\varphi_{0}=1.1 \arcsin \left(I / I_{\mathrm{dc}}\right) / N\right]$ takes to reach the intershell angular difference given by the stationary value [see Eq. (22)]. On the other hand, Eq. (27) for $I>I_{\mathrm{dc}}$ shows the intershell motion over the $-\pi / N<\varphi<\pi / N$ domain, which is indeed periodic. For $I \gg I_{\mathrm{dc}}$ Eq. (26) becomes $\tan (N \varphi / 2)=\zeta \approx \tan \left(\frac{N \varphi_{0}}{2}+\frac{\pi t}{T}\right)$; i.e., $\varphi=\varphi_{0}+2 \pi t / N T \sim$ $I\left(R_{+}^{-2}+R_{-}^{-2}\right) t$ becomes a linear function of time.

From the determined time dependence of $\varphi$ for $I>I_{\mathrm{dc}}$ we obtain the dynamics of each of the shells, by inserting Eq. (26) into Eqs. (16). Thereby, we have

$$
\omega_{ \pm}=\mp R_{ \pm}^{-2}\left(I-\frac{2 I_{\mathrm{dc}} \zeta}{1+\zeta^{2}}\right)
$$

where $\zeta$ is given by Eq. (26). For $I \leqslant I_{\mathrm{dc}}$ and $t \gg T, \omega_{ \pm}$goes asymptotically to 0 . For $I>I_{\mathrm{dc}}$, Fig. 4 shows the angular velocities of the vortex shell $\omega_{+}$[the time dependence of the antivortex shell $\omega_{-}$has opposite sign and differs only by a multiplication factor; see Eq. (29)] for the configuration $(0,3,-3)$ for $m_{0}=1.0$ and $z_{0}=0.05$. For these parameters we found $R_{-}=0.23368$ and $R_{+}=0.017206$. The angular velocities oscillate with frequency $1 / T$ [where the period $T$ is given by Eq. (28)]. For applied currents just above $I_{\mathrm{dc}}$ sharp peaks are observed. This is related to the shells being strongly coupled (moving with similar angular velocities) for a long time within 


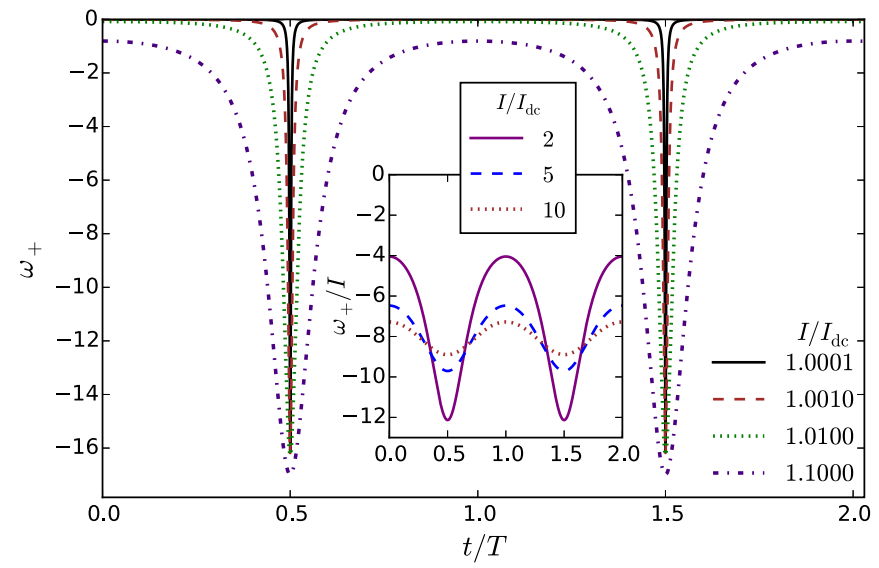

FIG. 4. Time dependence of the angular velocity $\omega_{+}$for the configuration $(0,3,-3)$, with $R_{-}=0.23368, R_{+}=0.017206$ (obtained for $m_{0}=1.0$ and $z_{0}=0.05$ ). Several values of $I>I_{\mathrm{dc}}$ are shown, with $\varphi(t=0)=\arcsin (1)$ as the initial condition. For $I \gtrsim I_{\mathrm{dc}}$ two shells move with almost same angular velocities, until sharp peaks of velocities in opposite directions appear with period $T$ [Eq. (28)]. (Inset) $\omega_{+} / I$ as a function of time. As $I$ is increased, sharp peaks transform into a sinusoidal behavior.

one oscillation before they tear apart. When this happens high angular velocities in opposite directions appear for a short time until the vortex and antivortex shells recouple and the process is repeated. As $I$ increases, the sharp peaks transform into a sinusoidal behavior (shown in the inset of Fig. 4), since $\varphi \approx 2 \pi t / N T$ and $\omega_{ \pm} \approx \mp R_{ \pm}^{-2}\left[I-I_{\mathrm{dc}} \sin (2 \pi t / T)\right]$ for $I \gg I_{\mathrm{dc}}$.

The shells' angular positions can be obtained by integrating Eq. (29) in time. For $I>I_{\mathrm{dc}}$, this yields

$$
\begin{aligned}
\varphi_{ \pm}(t)-\varphi_{ \pm}(0) & =\mp R_{ \pm}^{-2} \int d \varphi \frac{\left(I-I_{\mathrm{dc}} \sin N \varphi\right)}{\frac{d \varphi}{d t}} \\
& =\mp \frac{R_{\mp}^{2}}{\left(R_{+}^{2}+R_{-}^{2}\right)}\left[\varphi(t)-\varphi_{0}\right],
\end{aligned}
$$

where we assumed an external current constant in time and $\varphi$ given by Eqs. (25)-(27). Vortices have a larger angular displacement than antivortices, which is due to the increasing current density toward the center of the sample in the Corbino setup. The vortex and antivortex motions occur in short bursts for $I \gtrsim I_{\mathrm{dc}}$, while monotonically increasing with time for $I \gg I_{\mathrm{dc}}$. In the latter case the vortex shell's motion has an asymptotic behavior within one period of oscillation given by

$$
\varphi_{ \pm}(t)-\varphi_{ \pm}(0)=\mp \frac{2 \pi}{N} \frac{R_{\mp}^{2}}{\left(R_{+}^{2}+R_{-}^{2}\right)} \frac{t}{T} .
$$

Although the results of this section are derived for a thin superconducting disk, they are exact in the limit of an infinite film.

\section{INTERSHELL MOTION MODEL FOR COMMENSURATE VORTEX SHELLS}

In order to better understand the intershell dynamics for two shells of vortices (these shells can be constituted by either vortices or antivortices), we consider two vortex shells, 1 and
2, containing $N_{1}$ and $N_{2}$ vortices, respectively, such that $N_{1}=$ $(M-1) N_{2}$, where $M=1,2, \ldots$. Moreover, we assume all vortices in one shell are rotating with the same angular velocity $\omega_{\alpha=1,2}$. Therefore, after adding the contributions of all vortices within a given shell [see Eq. (A2)] we have the following set of equations describing the angular motion of the $\alpha$ th vortex shell,

$$
N_{\alpha}\left\langle R_{\alpha}^{2}\right\rangle \omega_{\alpha}=-\ell_{\alpha} I+\tau_{\alpha \beta},
$$

where $\ell_{\alpha}=\sum_{i=1}^{N_{\alpha}} v_{i}$ is the vorticity of the $\alpha$ th shell, $\left\langle R_{\alpha}^{2}\right\rangle=$ $\sum_{m=1}^{N_{\alpha}} R_{i}^{2} / N_{\alpha}$ is the mean square radial vortex position in this shell, and $\tau_{\alpha \beta}=\sum_{i=1}^{N_{\alpha}} \sum_{j=1}^{N_{\beta}} \tau_{i j}=-\tau_{\beta \alpha}$ is the torque on the $\alpha$ th shell due to the $\beta$ th shell. In the case where there are more than two shells one has to consider the torques exerted by all the $\beta \neq \alpha$ shells (the torques on the vortices due to other vortices in the same shell cancel out, since we consider a rigid shell). For the case of commensurate shells considered here the intershell torques are

$$
\begin{aligned}
\frac{\tau_{\alpha \beta}}{N_{2}}= & \sum_{m=1}^{M-1} \tau_{m, M}=-\sum_{m=1}^{M-1} \tau_{M, m}=-\frac{\tau_{\beta \alpha}}{N_{2}} \\
= & -2 v_{\alpha} v_{\beta} N_{2} R_{2}^{N_{2}} \sum_{m=1}^{M-1} R_{m}^{N_{2}}\left[\frac{1}{D_{N_{2}}\left(R_{m}, R_{2}, \varphi_{2, m}\right)}\right. \\
& \left.-\frac{1}{D_{N_{2}}\left(1, R_{m}, R_{2}, \varphi_{2, m}\right)}\right] \sin N_{2} \varphi_{2, m},
\end{aligned}
$$

where $v_{\alpha}\left(v_{\beta}\right)$ is the vorticity of the vortices in the $\alpha$ th ( $\beta$ th) shell, and $\varphi_{2, m}=\varphi_{2}-\phi_{m}$ is the angular separation between the vortex in shell 1 and one given vortex in shell 2 . Also, it is reasonable to consider the radial positions of the vortices in the less populated shell to be the same. However, even for commensurate shells the above expression for the intershell torques is still difficult to integrate analytically, since the vortices in shell 1 are not arranged in a regular polygon; i.e., they do not have the same radial positions and are not evenly spaced azimuthally. In fact, not only are the deviations of the vortex positions from the vertices of a regular polygon configuration dependent, they also depend on external parameters (such as, e.g., the magnetic dipole moment and position), as well as on the applied current $I$, since it changes the intershell separation. Consequently, the exact computation of the intershell torques requires the solution of Eqs. (A1) and (A2) for a given value of $I$.

Therefore, in order to derive a simple yet useful model for the intershell motion, instead of dealing with Eq. (32) combined with Eq. (33), we assume an intershell torque given by

$$
\tau_{\beta \alpha}=\tau_{\max } \sin N_{1} \varphi_{\alpha \beta} .
$$

Here $\tau_{\max }$ denotes the maximum torque one shell can exert on the other shell and it is directly related to the decoupling current $I_{\mathrm{dc}}$. Contrary to the case of equal number of vortices in the shells (which allow us to have an analytical formula for $I_{\mathrm{dc}}$ ), in this model the decoupling current becomes a parameter (which can be extracted from simulation data, for instance) [57]. Nonetheless, the above assumption simplifies 
considerably the intershell equation of motion, which becomes

$$
\begin{aligned}
\omega_{\alpha}-\omega_{\beta}= & \left(\frac{\ell_{\beta}}{N_{\beta}\left\langle R_{\beta}^{2}\right\rangle}-\frac{\ell_{\alpha}}{N_{\alpha}\left\langle R_{\alpha}^{2}\right\rangle}\right) I \\
& -\left(\frac{1}{N_{\alpha}\left\langle R_{\alpha}^{2}\right\rangle}+\frac{1}{N_{\beta}\left\langle R_{\beta}^{2}\right\rangle}\right) \tau_{\max } \sin N_{1} \varphi_{\alpha \beta} .
\end{aligned}
$$

This allows us to determine the dynamic behavior of the vortex configurations as a function of $I$, since Eq. (35) is similar to Eq. (23), with $\vartheta=N_{1} \varphi_{\alpha \beta}$, i.e.,

$$
N_{1}\left(\omega_{\alpha}-\omega_{\beta}\right)=\frac{d \vartheta}{d t}=K\left[I-I_{\mathrm{dc}} \sin \vartheta\right],
$$

where

$$
\begin{gathered}
K=N_{1}\left(\frac{\ell_{\beta}}{N_{\beta}\left\langle R_{\beta}^{2}\right\rangle}-\frac{\ell_{\alpha}}{N_{\alpha}\left\langle R_{\alpha}^{2}\right\rangle}\right), \\
I_{\mathrm{dc}}=\tau_{\max }\left(\frac{N_{\alpha}\left\langle R_{\alpha}^{2}\right\rangle+N_{\beta}\left\langle R_{\beta}^{2}\right\rangle}{\ell_{\beta} N_{\alpha}\left\langle R_{\alpha}^{2}\right\rangle-\ell_{\alpha} N_{\beta}\left\langle R_{\beta}^{2}\right\rangle}\right) .
\end{gathered}
$$

In Eqs. (37) and (38), specifically, $\alpha$ represents the outer shells. Therefore, we have the same solutions given by Eqs. (25)-(27), but with $N_{1}$ instead of $N$, and Eq. (28) becomes

$$
T=\frac{2 \pi}{K \sqrt{\left|I^{2}-I_{\mathrm{dc}}^{2}\right|}} .
$$

The angular velocities of each shell are found by plugging in Eqs. (32) the solution $\vartheta=N_{1} \varphi_{\alpha \beta}=2 \arctan [\zeta(t)]$ and by noticing that $\sin \vartheta=2 \tan (\vartheta / 2) /\left[1+\tan ^{2}(\vartheta / 2)\right]$ [Eq. (25)]. This yields

$$
\begin{aligned}
\omega_{\alpha} & =-\frac{1}{N_{\alpha}\left\langle R_{\alpha}^{2}\right\rangle}\left[\ell_{\alpha} I-\frac{2 \sigma_{\alpha \beta} I_{\mathrm{dc}} \zeta}{1+\zeta^{2}}\right] \\
& =\omega_{\mathrm{RB}}-\frac{\sigma_{\alpha \beta}}{N_{\alpha}\left\langle R_{\alpha}^{2}\right\rangle}\left(I-\frac{2 I_{\mathrm{dc}} \zeta}{1+\zeta^{2}}\right),
\end{aligned}
$$

where $\zeta(t)$ is given by Eqs. (25), (26), and (27), with $N_{1}$ instead of $N$,

$$
\sigma_{\alpha \beta}=\frac{\ell_{\alpha} N_{\beta}\left\langle R_{\beta}^{2}\right\rangle-\ell_{\beta} N_{\alpha}\left\langle R_{\alpha}^{2}\right\rangle}{N_{\alpha}\left\langle R_{\alpha}^{2}\right\rangle+N_{\beta}\left\langle R_{\beta}^{2}\right\rangle},
$$

and $\omega_{\mathrm{RB}}$ is the angular velocity the configuration would have if it was rotating as a RB [see Eq. (11) and taking into account that the vortex in the center, if present, has a negligible angular velocity in our model],

$$
\omega_{\mathrm{RB}}=-\frac{\left(\ell_{\alpha}+\ell_{\beta}\right) I}{N_{\alpha}\left\langle R_{\alpha}^{2}\right\rangle+N_{\beta}\left\langle R_{\beta}^{2}\right\rangle} .
$$

For the case of one shell with vortices and another with antivortices, we label the antivortex (vortex) shell index 2 (1). Therefore, $\ell_{2}=-N_{-}$and $\ell_{1}=N_{+}$, and the above equations become

$$
\omega_{ \pm}=\frac{\mp 1}{\left\langle R_{ \pm}^{2}\right\rangle}\left[I-\frac{N_{\mp}\left(\left\langle R_{+}^{2}\right\rangle+\left\langle R_{-}^{2}\right\rangle\right)}{N_{+}\left\langle R_{+}^{2}\right\rangle+N_{-}\left\langle R_{-}^{2}\right\rangle} \frac{2 I_{\mathrm{dc}} \zeta}{1+\zeta^{2}}\right] .
$$

For the case $N_{+}=N_{-}$we recover Eq. (29).
From the angular velocities we calculate the angular displacement of each shell as function of time. By performing similar algebraic manipulation as done for the case of zero total vorticity, we obtain

$$
\begin{aligned}
\Delta \varphi_{\alpha}(t) & =-\frac{\left(\ell_{\alpha}+\ell_{\beta}\right) I t}{N_{\alpha}\left\langle R_{\alpha}^{2}\right\rangle+N_{\beta}\left\langle R_{\beta}^{2}\right\rangle}-\frac{\sigma_{\alpha \beta} N_{1} \Delta \varphi_{\alpha \beta}(t)}{K N_{\alpha}\left\langle R_{\alpha}^{2}\right\rangle} \\
& =\omega_{\mathrm{RB}} t-\frac{\sigma_{\alpha \beta} N_{1} \Delta \varphi_{\alpha \beta}(t)}{K N_{\alpha}\left\langle R_{\alpha}^{2}\right\rangle},
\end{aligned}
$$

where $\Delta \varphi_{\alpha}(t)=\varphi_{\alpha}(t)-\varphi_{\alpha}(0)$ and $\Delta \varphi_{\alpha \beta}(t)=\varphi_{\alpha \beta}(t)-$ $\varphi_{\alpha \beta}(0)$. This equation shows that the shell displacement has an additional term, which depends linearly on the applied current, compared to the previous case of zero total vorticity [cf. Eq. (31)] and corresponds to the angular displacement of the RB frame rotating with angular velocity $\omega_{\mathrm{RB}}$.

For $I>I_{\mathrm{dc}}$ the case of a vortex and an antivortex shell presents more interesting features, since the attractive vortexantivortex interaction plays an important role in the intershell motion. Namely, the antivortex shell is dragged by the vortex shell (which experiences stronger current density being closer to the center of the disk) whenever individual vortices and antivortices are sufficiently close to each other. For low applied currents (just above $I_{\mathrm{dc}}$ ) this vortex-antivortex interaction prevails and the antivortex shell rotates alternately in the same and in the opposite direction to the rotation of the vortex shell. For higher currents, the induced Lorentz forces acting upon each shell overcome the vortex-antivortex interaction most of the time. Thereby, in this case, the shells rotate in opposite directions. This behavior is depicted in Fig. 5, where the angular displacements of the antivortex and vortex shells are shown as functions of time within one period $T$.

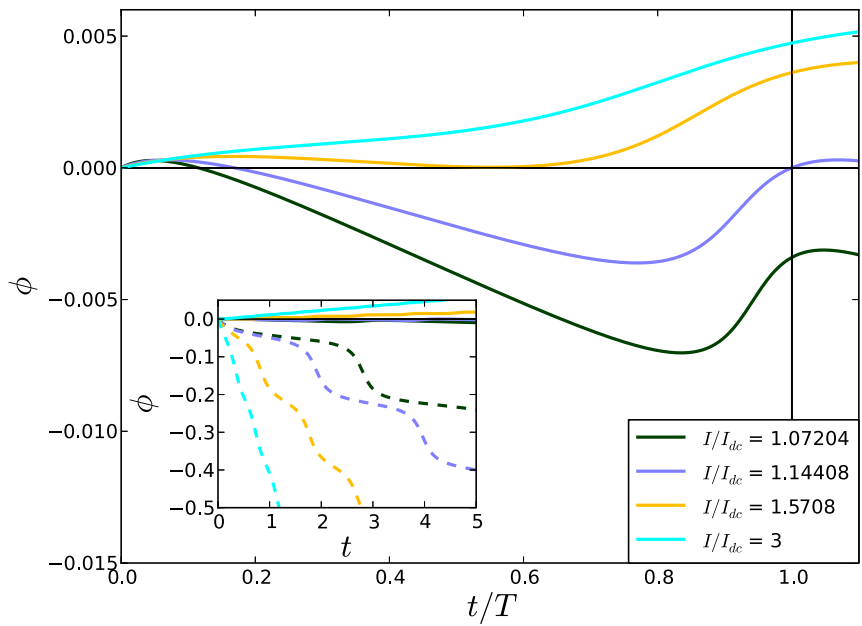

FIG. 5. Angular displacement of antivortices as a function of time (within one period $T$ ) for a configuration containing $N_{+}=6$ vortices and $N_{-}=3$ antivortices. The data were calculated for $\sqrt{\left\langle R_{+}^{2}\right\rangle}=0.0975, \sqrt{\left\langle R_{-}^{2}\right\rangle}=0.57$ and different values of $I>I_{\mathrm{dc}}$. The initial condition is $\varphi_{-}(t=0)=\varphi_{+}(t=0)=0$. For $I \gtrsim I_{\mathrm{dc}}$ the net angular displacement of antivortices is in the same direction as that of vortices (which move in the negative direction). For higher values of $I / I_{\mathrm{dc}}$ the antivortices rotate opposite to vortices. (Inset) Angular displacement of vortices (dashed lines) and antivortices (solid lines) as a function of time. 
For $I=1.07204 I_{\mathrm{dc}}$ the antivortex shell predominantly rotates in the same direction as the vortex shell. For $I \gtrsim 1.57 I_{\mathrm{dc}}$ the antivortex shell rotates only in the direction imposed by the applied current, even though the angular displacement is smaller whenever the vortices and antivortices come closer to each other. $I=1.14408 I_{\mathrm{dc}}$ is the threshold current for which the net antivortex angular motion is exactly zero. In the inset of Fig. 5 it is clear that the vortex and antivortex angular displacement increase with increasing applied current (this is not evidenced in the main panel since the period $T$ decreases for increased applied current).

The distinction between these two types of motion can be made by computing the net angular displacement of the antivortex shell during one period $T$. Therefore, making $t=T$ and $\Delta \varphi_{\alpha \beta}(T)=2 \pi / N_{1}$ and substituting Eq. (41) in Eq. (44), one obtains

$$
\begin{aligned}
\Delta \varphi_{\alpha}(T)= & -\frac{2 \pi}{K\left[N_{\alpha}\left\langle R_{\alpha}^{2}\right\rangle+N_{\beta}\left\langle R_{\beta}^{2}\right\rangle\right]} \\
& \times\left[\frac{\ell_{\beta} N_{\alpha}\left\langle R_{\alpha}^{2}\right\rangle-\ell_{\alpha} N_{\beta}\left\langle R_{\beta}^{2}\right\rangle}{N_{\alpha}\left\langle R_{\alpha}^{2}\right\rangle}-\frac{\left(\ell_{\alpha}+\ell_{\beta}\right) I}{\sqrt{I^{2}-I_{\mathrm{dc}}^{2}}}\right] .
\end{aligned}
$$

The threshold current $I^{*}$ where one of the shells changes the direction of rotation is given by the condition of zero net angular displacement within $T, \Delta \varphi_{-}(T)=0$. In this case the condition is fulfilled for

$$
\frac{1}{c_{\alpha \beta}}=\frac{\ell_{\alpha} N_{\beta}\left\langle R_{\beta}^{2}\right\rangle-\ell_{\beta} N_{\alpha}\left\langle R_{\alpha}^{2}\right\rangle}{N_{\alpha}\left\langle R_{\alpha}^{2}\right\rangle\left(\ell_{\alpha}+\ell_{\beta}\right)}=\frac{I^{*}}{\sqrt{I^{* 2}-I_{\mathrm{dc}}^{2}}},
$$

which yields

$$
I^{*}=\frac{I_{\mathrm{dc}}}{\sqrt{1-c_{\alpha \beta}^{2}}} .
$$

In the case where one of the shells contains antivortices, replacing $\alpha$ and $\beta$ with the antivortex and vortex shells labels - and + , respectively, we have

$$
c_{\alpha \beta}=c_{-+}=\frac{\left(N_{+}-N_{-}\right)\left\langle R_{-}^{2}\right\rangle}{N_{+}\left(\left\langle R_{+}^{2}\right\rangle+\left\langle R_{-}^{2}\right\rangle\right)} .
$$

Notice that $\left|c_{-+}\right|<1$, which yields $I^{*} \geqslant I_{\mathrm{dc}}$. Therefore, for $I<I^{*}$ the vortex-antivortex interaction is sufficiently strong to overcome the Lorentz force produced by the applied current, causing the net rotation of antivortices in the same direction as that of the vortices. On the other hand, for $I>I^{*}$ antivortices have net rotation opposite to vortices and in the direction dictated by the applied current. For the case depicted in Fig. 5, i.e., $N_{+}=6, N_{-}=3, \sqrt{\left\langle R_{+}^{2}\right\rangle}=0.0975$, and $\sqrt{\left\langle R_{-}^{2}\right\rangle}=0.57$, Eqs. (47) and (48) yield $I^{*}=1.14408 I_{\mathrm{dc}}$ for zero net antivortex motion. For the same number of vortices and antivortices in the shells $I^{*}=I_{\mathrm{dc}}$.

In Eq. (48) $c_{-+}$may also depend on $I_{\mathrm{dc}}$, which results in a nonlinear dependence of $I^{*}$ on $I_{\mathrm{dc}}$. This is so because $I_{\mathrm{dc}}$ depends on the vortex and antivortex positions, so it may depend on $\left\langle R_{-}^{2}\right\rangle$ and $\left\langle R_{+}^{2}\right\rangle$ as well. Conversely, one can think of $\left\langle R_{-}^{2}\right\rangle$ and $\left\langle R_{+}^{2}\right\rangle$ depending on $I_{\mathrm{dc}}$. So, for $N_{+}>N_{-}, c_{-+}$might depend on $I_{\mathrm{dc}}$. However, in our case we expect this dependence to be small since $c_{-+}=\left(1-N_{-} / N_{+}\right) /\left(1+\left\langle R_{+}^{2}\right\rangle /\left\langle R_{-}^{2}\right\rangle\right) \approx$ $\left(1-N_{-} / N_{+}\right)$because typically we have $\sqrt{\left\langle R_{+}^{2}\right\rangle /\left\langle R_{-}^{2}\right\rangle}$ between 0.2 and 0.25 . For the case $N_{+}=6, N_{-}=3, \sqrt{\left\langle R_{+}^{2}\right\rangle}=$ 0.0975 , and $\sqrt{\left\langle R_{-}^{2}\right\rangle}=0.57$, making $c_{-+}=\left(1-N_{-} / N_{+}\right)$ gives $I^{*}=1.155 I_{\mathrm{dc}}$, which is a very good approximation to the exact value.

It is interesting to point out that in the case of both shells being constituted by vortices there is no real value for $I^{*}$, since $\left|c_{\alpha \beta}\right|>1$. This should be so since in this case the shells are expected to rotate always in the same direction when driven by the external current.

\section{VOLTAGE-CURRENT CHARACTERISTICS}

Measurements of the voltage-current characteristics is a standard experimental manner to examine the dynamic regimes of vortices in the Corbino geometry. For the case of two-shell dynamics, a suitable arrangement of voltage contacts $a, b$, and $c$, as illustrated schematically in Fig. 6, allows one to probe the motion of each shell individually by measuring the voltage drop between two point contacts. In the case depicted in Fig. 6, the voltage corresponding to the first (second) vortex shell would be $V_{1}=V_{a b}\left(V_{2}=V_{b c}\right)$. The overall response of the system is simply the sum of these voltages.

Whenever a vortex passes between two voltage contacts a voltage pulse $V_{i}(t)$ is detected. As pointed out by Clem [58], irrespectively of the pulse shape, its time integral is $\int V_{i}(t) d t=\Phi_{0}$. In the Corbino geometry, a single vortex or antivortex will generate $N_{r}$ such pulses, where $N_{r}$ is the number of revolutions performed during the measurement time $\Delta t_{\text {exp. }}$. The pulses will be positive if the (anti)vortex moves (counter)clockwise or negative otherwise. Notice that, for an intricate dynamics, a vortex may pass back and forth through the line between the contacts, thus inducing both

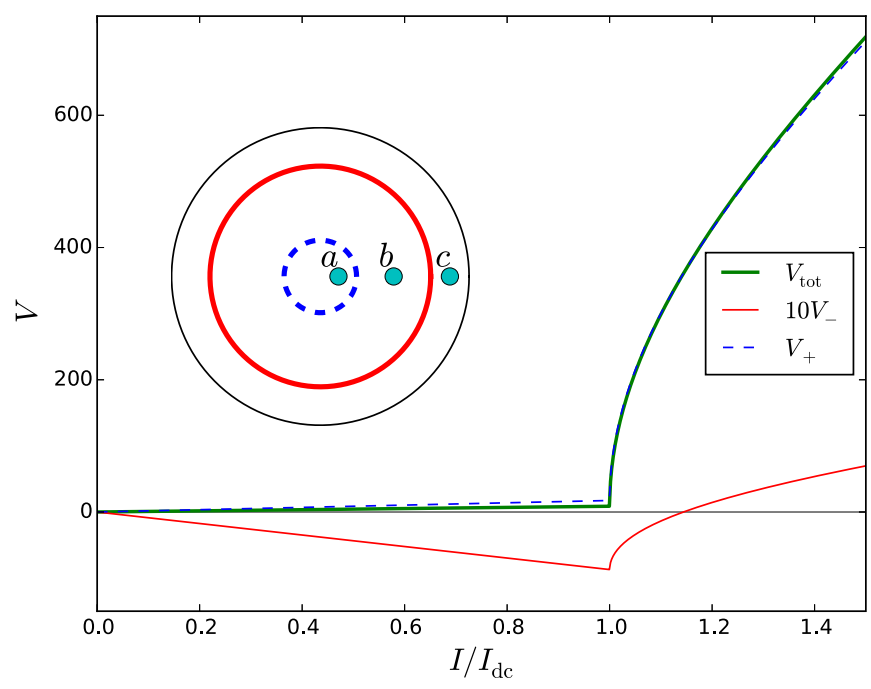

FIG. 6. Time-averaged voltage associated with the antivortex shell (red), vortex shell (blue), and configuration (green) motion as function of the applied current for the same configuration shown in Fig. 5. (Inset) Scheme of three point contacts (light blue dots) between which the vortex shells may move (depicted as large red and blue circles). 
negative and positive pulses. These obviously cancel out after a long measurement time. In the end, what matters is the total angular (counter)clockwise displacement of the vortex $\Delta \phi_{i}= \pm 2 \pi N_{r}=\Omega_{i} \Delta t$, where $\Omega_{i}=\frac{1}{\Delta t_{\exp }} \int_{\Delta t_{\exp }} \omega_{i}(t) d t$ is the time-averaged angular velocity of the vortex. Therefore, for a single vortex, one can write

$$
V_{i}=-v_{i} \frac{\Phi_{0}}{2 \pi} \Omega_{i}
$$

Assuming that during $\Delta t_{\exp }$ the vortex shell $\alpha$ keeps its integrity (that is, $N_{\alpha}$ is constant and vortices do not overpass each other), all vortices in the shell will perform the same number of revolutions. Therefore, the contribution of each vortex is the same and the voltage drop due to shell rotation reads

$$
V_{\alpha}=-\ell_{\alpha} \frac{\Phi_{0}}{2 \pi} \Omega_{\alpha}
$$

where $\Omega_{\alpha}$ is the time-averaged angular velocity of the shell and $\ell_{\alpha}=v_{\alpha} N_{\alpha}$ is its total vorticity.

For the commensurate shell motion considered here, $\omega_{\alpha}(t)$ is a periodic function with a period $T$ given by Eq. (39). In this case, the average over $\Delta t_{\exp }$ may be replaced by the average over $T$.

Substituting Eqs. (40)-(42) in Eq. (50) above and performing the required time integration, we find [in units of $\left.\Phi_{0} /\left(2 \pi t_{0}\right)\right]$

$$
\begin{aligned}
V_{\alpha}= & \frac{\ell_{\alpha}}{N_{\alpha}\left\langle R_{\alpha}^{2}\right\rangle+N_{\beta}\left\langle R_{\beta}^{2}\right\rangle}\left[\left(\ell_{\alpha}+\ell_{\beta}\right) I\right. \\
& \left.+\left(\frac{\ell_{\alpha} N_{\beta}\left\langle R_{\beta}^{2}\right\rangle}{N_{\alpha}\left\langle R_{\alpha}^{2}\right\rangle}-\ell_{\beta}\right) \sqrt{I^{2}-I_{\mathrm{dc}}^{2}} \Theta\left(I-I_{\mathrm{dc}}\right)\right],
\end{aligned}
$$

where $\Theta(x)$ is the Heaviside step function. The first term is proportional to $\omega_{\mathrm{RB}}$, which varies linearly with $I$ and, therefore, is Ohmic. The other contribution, for $I>I_{\mathrm{dc}}$ comes from the relative motion between the shells. For the specific case where there are commensurate shells of vortices and antivortices, we have

$$
\begin{aligned}
V_{\mp}= & \frac{N_{\mp}}{N_{-}\left\langle R_{-}^{2}\right\rangle+N_{+}\left\langle R_{+}^{2}\right\rangle}\left[\mp\left(N_{+}-N_{-}\right) I\right. \\
& \left.+N_{ \pm}\left(\frac{\left\langle R_{ \pm}^{2}\right\rangle}{\left\langle R_{\mp}^{2}\right\rangle}+1\right) \sqrt{I^{2}-I_{\mathrm{dc}}^{2}} \Theta\left(I-I_{\mathrm{dc}}\right)\right],
\end{aligned}
$$

while in the case of configurations with only vortices (considering $N_{\alpha}=N_{1}>N_{\beta}=N_{2}, R_{1}>R_{2}$ ), we find

$$
\begin{aligned}
V_{(1,2)}= & \frac{N_{(1,2)}}{N_{1}\left\langle R_{1}^{2}\right\rangle+N_{2}\left\langle R_{2}^{2}\right\rangle}\left[\left(N_{1}+N_{2}\right) I\right. \\
& \left.+N_{(2,1)}\left(\frac{\left\langle R_{(2,1)}^{2}\right\rangle}{\left\langle R_{(1,2)}^{2}\right\rangle}-1\right) \sqrt{I^{2}-I_{\mathrm{dc}}^{2}} \Theta\left(I-I_{\mathrm{dc}}\right)\right] .
\end{aligned}
$$

In the latter case the initial Ohmic response starts to increase (decrease) with $\sqrt{I^{2}-I_{\mathrm{dc}}^{2}}$ for currents above $I_{\mathrm{dc}}$ for the voltage associated with the shell 2 (1).
On the other hand, for configurations with vortices and antivortices, for $I<I_{\mathrm{dc}}$ the Ohmic response has opposite voltages due to the motion of vortices and antivortices in the same direction (RB motion of the configuration). For $I>I_{\mathrm{dc}}$, there is an additional positive contribution proportional to $\sqrt{I^{2}-I_{\mathrm{dc}}^{2}}$. While the voltage associated with vortices always has the same sign, the one due to antivortex motion changes sign. From Eq. (52) one verifies that the applied current at which this voltage changes sign is given by Eqs. (47) and (48). This is expected, since the latter equations describe the current at which the antivortex shell does not have any net motion; therefore, $V_{-}\left(I^{*}\right)=0$.

Figure 6 shows $V_{+}, V_{-}$, and $V_{\text {tot }}=V_{+}+V_{-}$[divided by $\left.V_{\mathrm{tot}}\left(I_{\mathrm{dc}}\right)\right]$ as a function of the applied current for the $(1,6,-3)$ configuration with $R_{+} \approx 0.0975$ and $R_{-} \approx 0.57$ (the same values used to plot Fig. 5). The voltages depict the behavior mentioned above: For $I<I_{\mathrm{dc}}$ the linear response is related to the RB motion of the entire configuration, while for $I>I_{\mathrm{dc}}$ a contribution proportional to $\sqrt{I^{2}-I_{\mathrm{dc}}^{2}}$ is obtained [59]. Notice also that for $I<I_{\mathrm{dc}}$ the voltage produced by the moving antivortices is opposite in sign to the one produced by moving vortices, since they move in the same direction and the electric fields generated by vortex and antivortex shells have opposite sign. In addition, in the range $I_{\mathrm{dc}}<I<I^{*}, V_{-}$still has opposite sign to $V_{+}$because the net motion of the antivortices is in the same direction as the vortex shell rotation. Nevertheless, $V_{\text {tot }}$ has the same sign as $V_{+}$for in the whole range of the applied current.

We also computed the power dissipated due to the vortex motion. The dissipated power $\mathcal{P}$ in a given volume, $V$, is given by $\int_{V} d^{3} r \mathbf{E} \cdot \mathbf{j}$, where $\mathbf{E}$ is the electric field and $\mathbf{j}$ is the current density. Following the Bardeen-Stephen approximation, we consider the electric fields only inside the vortex cores, given by Eq. (49). Therefore, for $N_{S}$ shells, each moving with angular velocity $\omega_{\alpha}$ and containing $N_{\alpha}$ vortices or antivortices, we have

$$
\begin{aligned}
\mathcal{P} & \approx \pi \xi^{2} \sum_{i=1}^{N_{v}} \frac{v_{i} \Phi_{0}\left(\hat{z} \times \mathbf{v}_{i}\right)}{\pi \xi^{2}} \cdot \mathbf{J}\left(\mathbf{r}_{i}\right) \\
& =\sum_{i=1}^{N_{v}} \mathbf{v}_{i} \cdot \mathbf{F}_{i}=\sum_{i=1}^{N_{v}} \mathbf{v}_{i} \cdot \mathbf{v}_{i}=\sum_{i=1}^{N_{v}}\left(\omega_{i}^{2} r_{i}^{2}+v_{i, r}^{2}\right) \\
& \approx \sum_{\alpha=1}^{N_{S}} N_{\alpha}\left\langle R_{\alpha}^{2}\right\rangle \omega_{\alpha}^{2} .
\end{aligned}
$$

Here $\left\langle R_{\alpha}^{2}\right\rangle=\sum_{i=1}^{N_{\alpha}} r_{i}^{2} / N_{\alpha}, \mathbf{F}_{i}$ is the force acting on the $i$ th vortex, and we used the Bardeen-Stephen equation of motion, $\mathbf{v}_{i}=\mathbf{F}_{i}$. For the case of two commensurate shells, integrating the above equations over one period, $T$, results in the timeaveraged dissipated power

$$
\begin{aligned}
\langle\mathcal{P}\rangle & =\left(N_{\alpha}\left\langle R_{\alpha}^{2}\right\rangle\left\langle\omega_{\alpha}^{2}\right\rangle_{T}+N_{\beta}\left\langle R_{\beta}^{2}\right\rangle\left\langle\omega_{\beta}^{2}\right\rangle_{T}\right) \\
& =\left[R_{\mathrm{RB}}+\rho_{\mathrm{s}} \sqrt{\left.1-\frac{I_{\mathrm{dc}}^{2}}{I^{2}} \Theta\left(I-I_{\mathrm{dc}}\right)\right] I^{2},}\right.
\end{aligned}
$$


where

$$
\begin{gathered}
R_{\mathrm{RB}}=\frac{\left(\ell_{\alpha}+\ell_{\beta}\right)^{2}}{N_{\alpha}\left\langle R_{\alpha}^{2}\right\rangle+N_{\beta}\left\langle R_{\beta}^{2}\right\rangle}, \\
\rho_{\mathrm{s}}=\left(\frac{1}{N_{\alpha}\left\langle R_{\alpha}^{2}\right\rangle}+\frac{1}{N_{\beta}\left\langle R_{\beta}^{2}\right\rangle}\right) \sigma_{\alpha \beta}^{2} .
\end{gathered}
$$

Substituting Eq. (41) in the above equations we find $\langle\mathcal{P}\rangle=$ $\left(V_{\alpha}+V_{\beta}\right) I$, where $V_{\alpha}$ and $V_{\beta}$ are given by Eq. (51), as should be expected.

\section{SIMULATION RESULTS}

In addition to the analytic model detailed in the previous sections, we performed molecular dynamics simulations of vortices in a thin disk of radius $R$ in both scenarios: (i) with a magnetic dipole on top (as illustrated in Fig. 1) and (ii) subjected to a homogeneous magnetic field $H_{a}$. For both cases, the initial vortex configurations were obtained by integrating Eq. (1) for $I=0$ using the method described in Refs. [38,60] for different values of the control parameters $m_{0}$ and $z_{0}$ for the dipole case and $H_{a}$ for the homogeneous field case. Then, for each configuration, a radial current $I$ was gradually increased from $I=0$, taking as initial configuration the one obtained for the previous value of current. For each current, the vortex dynamics was recorded for more than $10^{6}$ time steps. In all simulations, the intervortex potential was cut off inside the vortex cores.

The simulation results reveal the same general trends predicted by our analytical model. At low currents, the system behaves as a RB, with both shells rotating in the same direction with the same angular velocities. Above a decoupling current, $I_{\mathrm{dc}}$, the shells decouple and rotate with different angular velocities in a highly correlated motion. In the case of the disk with a magnetic dipole on top, the antivortex and vortex shells rotate in opposite directions at an even higher current (see also Ref. [52]). For all configurations under investigation here, and in both scenarios, the RB angular velocity was found to follow Eq. (11) exactly. In fact, since Eq. (11) is a direct result of the the RB constraint, one can use it to check whether the simulations are running properly. However, all other results of our analytic model rely on assumptions such as shells forming rigid, regular polygons. In what follows, we check thoroughly in which extent these approximations represent an accurate description of the dynamics of two vortex shells in superconducting disks.

\section{A. The rigid-body phase}

We first compare our analytical results to the numerical ones for the moving RB phase. We start with the case of the dipoledisk system with equally populated shells $\left(N_{+}=N_{-}\right)$. In this case, a key feature of the model is that the steady-state value of the angular displacement between both shells increases with the current as $\arcsin \left(I / I_{\mathrm{dc}}\right)$ [see Eq. (24)] as a result of the shear gradient induced by the $1 / r$ behavior of the current density. To check this, we computed the quantity $\Delta \phi=\varphi_{-+}(I)-\varphi_{-+}(0)$, which represents the difference between the angular separation of each vortex of the inner shell to the nearest antivortex in the outer shell at a current value $I$ compared to $I=0$ for different

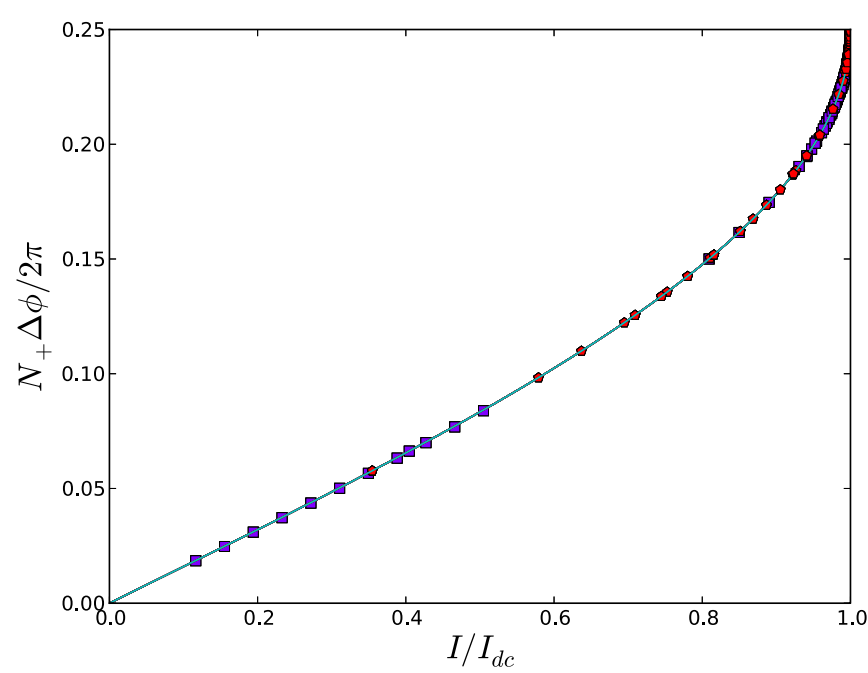

FIG. 7. Vortex-antivortex angular separation, $\Delta \phi$, as a function of the applied current $I$ in the RB phase for the states $(1,4,-4)$ (squares) and $(1,5,-5)$ (pentagons). The $(1,4,-4)$ state was obtained for $m_{0}=2.4$ and $z_{0}=0.06,0.07, \ldots, 0.1$; and $m_{0}=$ $2.6,2.8, \ldots, 3.8$ and $z_{0}=0.1$. The $(1,5,-5)$ state was obtained for $m_{0}=3.2$ and $z_{0}=0.09,0.1 ; m_{0}=3.4$ and $z_{0}=0.1 ;$ and $m_{0}=3.6$ and $z_{0}=0.08,0.09$. The solid line depicts the theoretical dependence $N_{+} \Delta \phi=\arcsin \left(I / I_{d c}\right)$ provided by our analytic model. $I$ is normalized to the corresponding decoupling current of the considered state.

shell configurations. In Fig. 7 we show $N_{+} \Delta \phi$ as a function of $I / I_{\mathrm{dc}}$ for the states $(1,4,-4)$ and $(1,5,-5)$ obtained for different values of $m_{0}$ and $z_{0}$. Regardless of the state and the chosen $m_{0}$ and $z_{0}$, all these data collapse on one curve and are fitted remarkably well by the theoretical dependence $N_{+} \Delta \phi=\arcsin \left(I / I_{\mathrm{dc}}\right)$.

The angular displacements for configurations having commensurate (but not equal) shell occupation in the dipole-disk system are shown in Fig. 8. The configurations were obtained for different values of $m_{0}$ and $z_{0}$. Since for each antivortex there are $N_{+} / N_{-}$vortices in the inner shell (with $N_{+} / N_{-}$ varying from 2 to 6$), N_{+} / N_{-}$values of $\Delta \phi$ are found for each configuration. The dependence of $\Delta \phi$ on $I / I_{\mathrm{dc}}$ is roughly the same for all the vortices and can be described well by the functional dependence $N_{+} \Delta \phi=\arcsin \left(I / I_{\mathrm{dc}}\right)+2 \pi n$, where $n=0,1, \ldots, N_{+} / N_{-}-1$. This indicates that our ansatz [Eq. (34)] for the intershell torque works as a good approximation for these configurations. However, in contrast to the $N_{+}=N_{-}$case, some discrepancies from the predicted behavior are apparent. That is because, in general, the shells do not form regular polygons, contrary to the assumption taken in our analytic model assumption. If we denote by $R_{+}+\delta R_{n}$ and $2 \pi n / N_{+}+\delta \phi_{n}$ the radial and angular positions of the $n$th vortex (for $I=0$ ) we find that $\delta R_{n}$ and $\delta \phi_{n}$ grows as $N_{+} / N_{-}$increases. This feature remains also for nonzero applied current and can be seen in Fig. 8 .

For the case where the disk is subjected to a homogeneous field, the stable configurations contain only vortices and, in general, there are fewer vortices in the inner shell $\left(N_{\text {int }}\right)$ than in the outer one $\left(N_{\text {out }}\right)[36,38,61]$. In this case, the model developed in Sec. IV predicts an angular displacement [in relation to $\phi(0)] \Delta \phi=\arcsin \left(I / I_{\mathrm{dc}}\right) / N_{\text {out }}$ for commensurate 


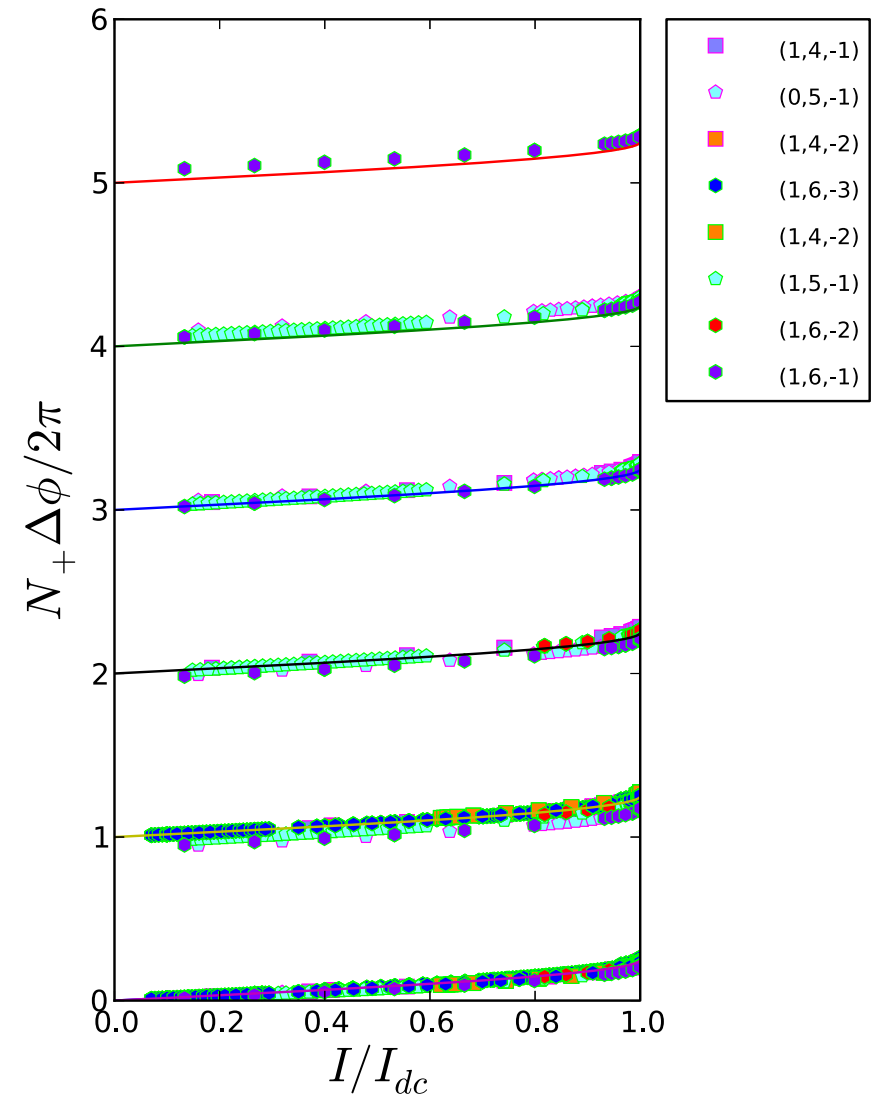

FIG. 8. Vortex-antivortex angular separation, $\Delta \phi$, as a function of $I / I_{\mathrm{dc}}$ in the RB phase for several configurations with commensurate inner (vortex) and outer (antivortex) shells. The symbols with green and red edges represent data obtained for $m_{0}=8.0$ and $m_{0}=6.0$, respectively. The state $(1,6,-3)$ contains data calculated for $z_{0}=0.2,0.21$, and 0.22 . The solid lines depict the $N_{+} \Delta \phi=$ $\arcsin \left(I / I_{\mathrm{dc}}\right)+2 \pi n$ dependence, where $n=0,1, \ldots, N_{+} / N_{-}-1$, expected by our model. Due to the shells' commensurability there are $N_{-}$antivortices with $N_{+} / N_{-}$angular separations to corresponding nearest vortices.

shells. Figure 9(e) shows the good agreement between this prediction and the simulation results for states $(0,3,9)\left(H_{a}=\right.$ 16.0), $(0,4,12)\left(H_{a}=24.0\right)$, and $(1,6,12)\left(H_{a}=27.0\right)$, depicted in Figs. 9(a), 9(b), and 9(d), respectively. For $(0,6,12)$ $\left(H_{a}=27.0\right)$, shown in Fig. 9(c), we find a similar dependence to our model, except that, instead of $N_{\text {out }}$ in the above equation, one has $\sim 0.75 N_{\text {out }}$. This difference between predicted and fitted values seems to be related to the inner shell geometry, which is closer to a deformed triangle instead of a regular hexagon. In this configuration the angular separations between neighboring vortices in the inner shell are equal to $\pi / 3$ for all the vortices (as it would be for a regular hexagon), but the radial distances are not. In fact, the inner shell is best described by two equilateral triangles of different sizes.

Another observed feature is that the angular separation $\Delta \phi(I)$ is not exactly the same for all vortices in the outer shell. This is more pronounced for the $(0,6,12)$ configuration, but it is also present in the other configurations depicted in Fig. 9. This indicates that the outer shell would be better described as $N_{\text {out }} / N_{\text {in }}$ polygons with $N_{\text {in }}$ sides. However, our model of rigid
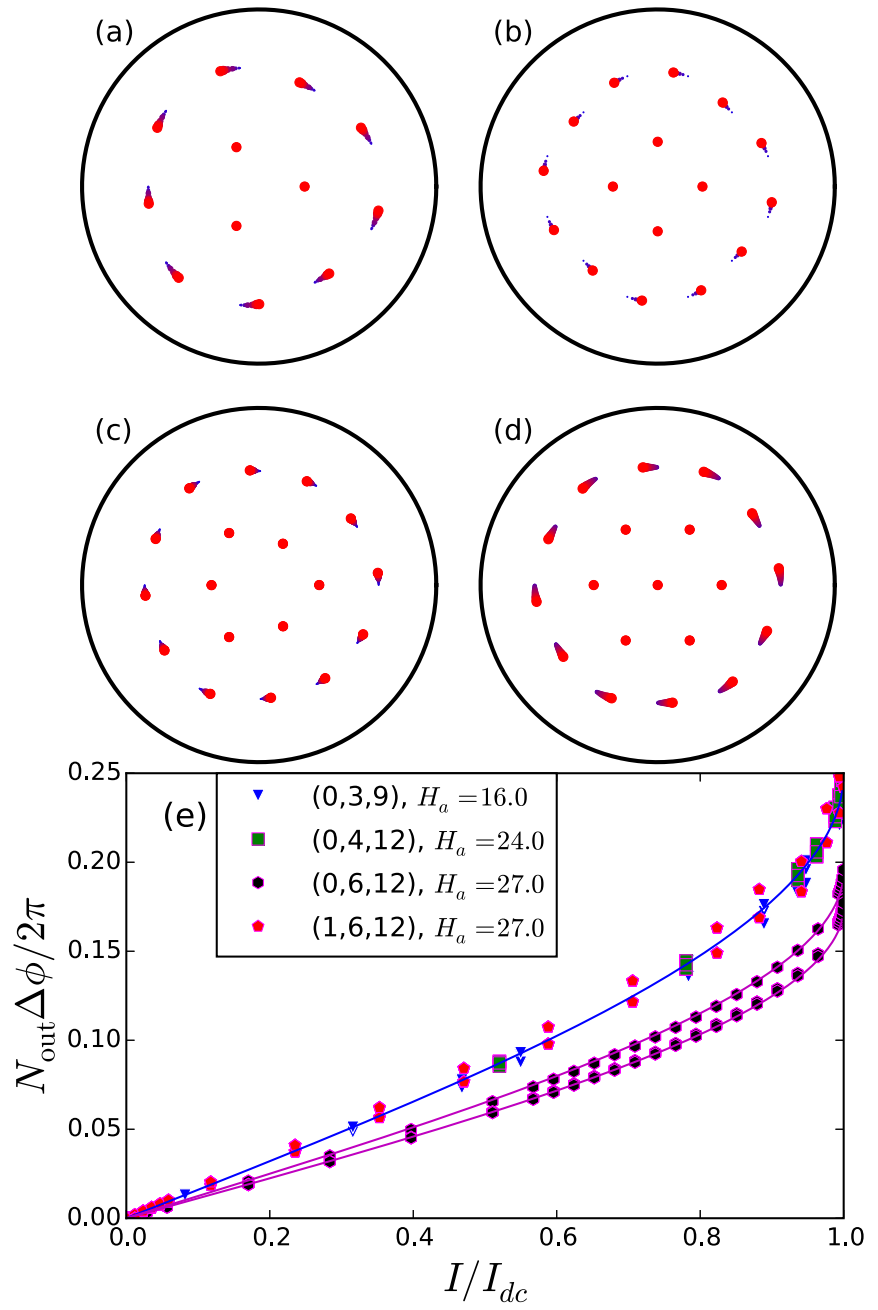

FIG. 9. Vortex positions for weak applied currents and states (a) $(0,3,9)$ at $H_{a}=16.0,(\mathrm{~b})(0,4,12)$ at $H_{a}=24.0$, (c) $(0,6,12)$ at $H_{a}=27.0$, and (d) $(1,6,12)$ at $H_{a}=27.0$ in the frame rotating with angular velocity of the inner shell for $I<I_{\mathrm{dc}}$. The vortex markers are made larger as the applied current increases. (e) The angular displacements between vortices in the inner and outer shells $\Delta \phi$ as a function of the applied current $I$ [corresponding to the configurations shown in panels (a)-(d)]. The blue line depicts the analytic equation $\Delta \phi=\arcsin \left(I / I_{\mathrm{dc}}\right) / N_{\text {out }}$, while the magenta lines represent $\Delta \phi=$ $\arcsin \left(I / I_{\mathrm{dc}}\right) / N^{\prime}$, where $N^{\prime}=0.7 N_{\text {out }}$ and $N^{\prime}=0.78 N_{\text {out }}$.

outer and inner vortex shells is still a reasonable approximation in those cases.

\section{B. The decoupled-shells phase}

In what follows we analyze the more intricate dynamics of the decoupled phase $\left(I>I_{\mathrm{dc}}\right)$. A salient feature of this phase is the periodic sequence of detachments and reconnections as the shells move relative to each other. These are reflected in the time evolution of the shells' angular velocities as a series of peaks with a well-defined period $T$. This behavior, already predicted by our analytic model (cf. Fig. 4), is illustrated in Fig. 10 for the configuration $(1,4,-4)$. The simulation data are in excellent agreement with the analytical prediction obtained from Eq. (18). Notice that the influence of the vortex 


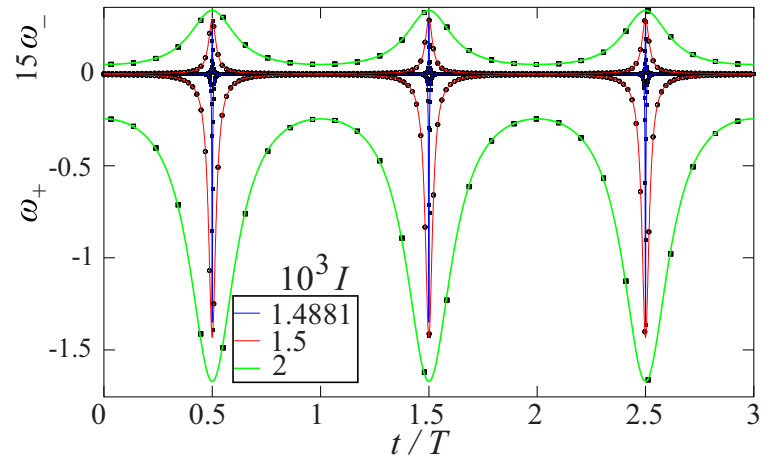

FIG. 10. Vortex and antivortex angular velocities, $\omega_{+}$and $\omega_{-}$, respectively, as a function of time, for the configuration $(1,4,-4)$ (for $\left.m_{0}=3.0, z_{0}=0.1\right)$ and different applied currents. Points and lines represent the simulation data and the analytical results, respectively. For the above analytical curves we used $I_{\mathrm{dc}}=1.4880275 \times 10^{-3}$.

in the center of the disk on the intershell dynamics is negligible, since its radial position is very close to zero.

In the model developed for equally populated shells, the frequency of reconnection of the shells is given by $f=1 / T=$ $(K / 2 \pi) \sqrt{I^{2}-I_{\mathrm{dc}}^{2}}$, where $K$ is a function of the shell radii [see Eq. (28)]. To check the validity of this equation, we calculated the reconnection frequency, normalized by $f_{N}=I_{\mathrm{dc}} K / 2 \pi$, as a function of $I / I_{\mathrm{dc}}$ for configurations $(1,4,-4)$ and $(1,5,-5)$ and different values of $m_{0}$ and $z_{0}$ (which result in different values of the shell radii $R_{+}$and $R_{-}$). The results, presented in Fig. 11(a), reveal a remarkable collapse of all numerical data points precisely on the predicted theoretical curve. Figure 11(b) shows the dependence of the decoupling current $I_{\mathrm{dc}}$, calculated numerically, on the ratio $R_{+} / R_{-}$for the configurations $(1,4,-4)$ and $(1,5,-5)$ for the same values of $m_{0}$ and $z_{0}$ used in Fig. 11. Once more, the numerical data are in excellent agreement with the theoretical prediction, given by Eq. (21).

Similar series of detachments and reconnections can be observed for the case where the vortex and the antivortex shells in the disk-dipole system are commensurate, but not equally populated. Figure 12 shows the angular velocities of the shells as functions of time for the configuration $(1,6,-2)$. For a better visualization, the angular velocities were divided by the corresponding current and time was normalized by the theoretical value of the period $T$ [calculated from Eq. (39) with $N_{+}$instead of $N_{1}$ ]. Points and lines depict the results of the simulation and the model, respectively. Once again, a very good agreement between the numerical data and the analytic prediction is observed. The same conclusion extends to other commensurate configurations we have analyzed, $(1,4,-2)$ and $(1,6,-3)$, as well as for a particular weakly commensurate configuration $(1 g 2,6,-4)$ (from here on, $1 g n$ stands for a giant vortex of vorticity $n$ located at the center). In all these cases, the shells form polygons very close to regular and the central vortex radial position is practically zero. However, for other weakly commensurate configurations, such as $(1,4,-1),(1,6,-1),(1 g 2,5,-2)$, and $(1 g 3,4,-3)$, the shells deform considerably from regular polygons and the displacement of the central (giant) vortex from the disk center is appreciable. For these configurations, the quantitative
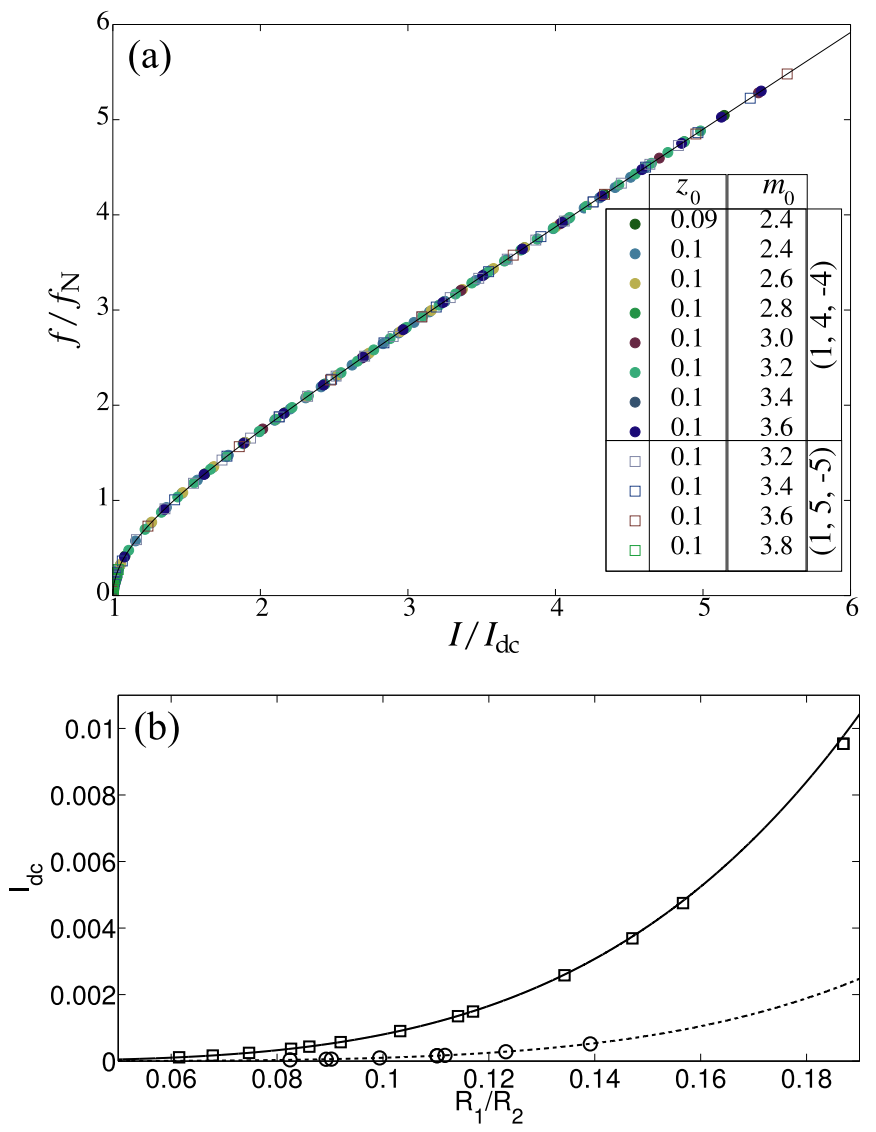

FIG. 11. (a) Normalized reconnection frequency $f / f_{N}\left(f_{N}=\right.$ $\left.K I_{\mathrm{dc}} / 2 \pi\right)$ of configurations $(1,4,-4)$ and $(1,5,-5)$ as a function of normalized current $\left(I^{\prime}=I / I_{\mathrm{dc}}\right)$ calculated for different values of the control parameters $m_{0}$ and $z_{0}$. (b) Decoupling current $I_{\mathrm{dc}}$ as a function of the reduced radial position $R_{+} / R_{-}$extracted from the simulations considered in (a). The continuous and dashed lines are plots of Eq. (21) for $N_{+}=N_{-}=4$ and $N_{+}=N_{-}=5$, respectively.

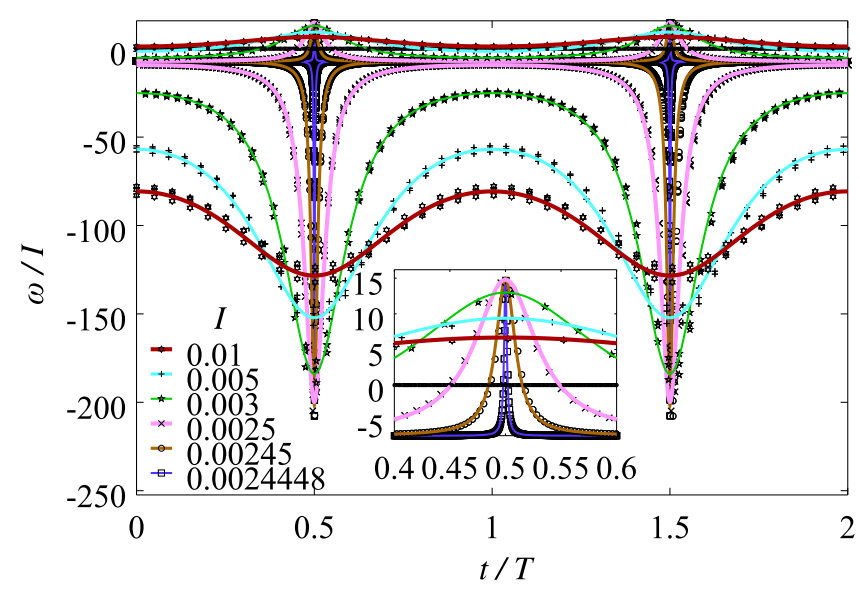

FIG. 12. Time dependence of the vortex and the antivortex angular velocities for the configuration $(1,6,-2), m_{0}=8.0, z_{0}=$ 0.2 and different applied currents $\left(I_{\mathrm{dc}}=2.4447 \times 10^{-3}\right.$ gives best analytical fit to the simulation results). Points (lines) depict the results from the simulation (model). The angular velocities were divided by the applied current for better visualization. (Inset) Magnification of the peaks in the angular velocity for the antivortex shell. 


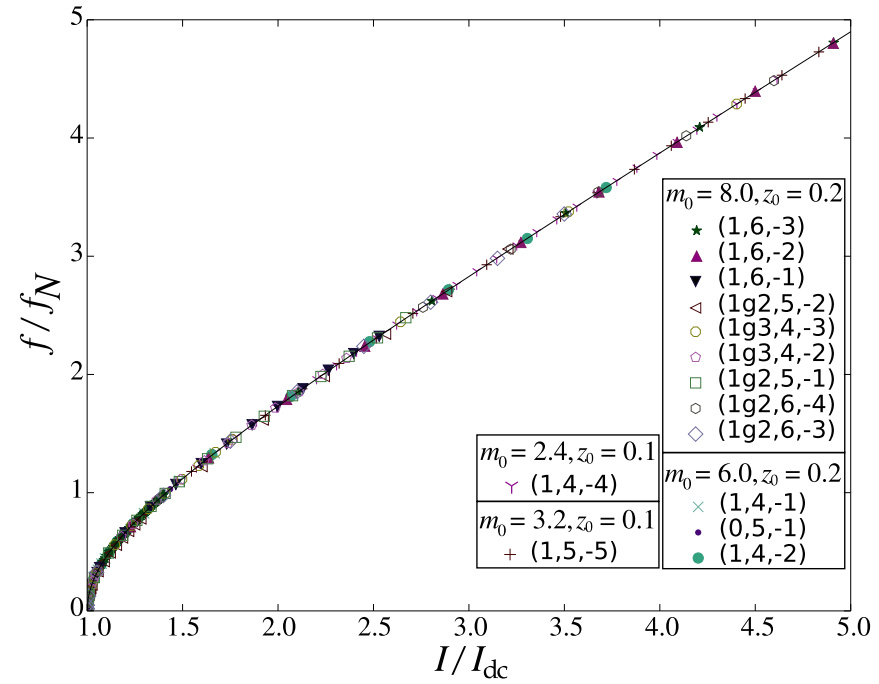

FIG. 13. The dependence of $f / f_{N}$ on $I / I_{\mathrm{dc}}$ for all of the investigated vortex-antivortex configurations.

agreement between the model and the simulations is much poorer, though all qualitative trends remain captured by the model.

The functional dependence of the frequency of the peaks in angular velocity on the applied current was found to be the same for all the investigated configurations. In fact, Fig. 13 shows that all of them can be adjusted to follow the same curve, that agrees with the curves for the configurations $(1,4,-4)$ and $(1,5,-5)$ discussed before [see Fig. 11(a)]. Therefore, although not providing a prediction of $I_{\mathrm{dc}}$ for the cases where $N_{1} \neq N_{2}$, the functional dependence predicted by the model is the same as the one found in simulations even in this case. Moreover, the fitting parameter $f_{N}$ obtained for commensurate configurations with $N_{-}>1$ shows a good agreement with Eq. (28).

Finally, we test the results of our model in the decoupled phase for the case of a disk subjected to a homogeneous field. As expected, the time evolution of the angular velocity of each shell also presents peaks associated with the slippage of the vortices in one shell over the vortices in the other shell. Figure 14 shows that the reconnection frequency of all configurations considered in our simulations follow the $\sqrt{I^{2}-I_{\mathrm{dc}}^{2}}$ law predicted by our model [see Eq. (39)]. However, contrary to the case with vortices and antivortices, in the present situation the normalization factor $f_{N}$ differs from the predicted one, $f_{N}^{\text {model }}=K I_{\mathrm{dc}} / 2 \pi$, where $K$ is given by Eq. (37). In fact, we find for $(0,3,9) f_{N}=$ 0.1277 and $f_{N}^{\text {model }}=0.1573$; for $(0,4,10) f_{N}=0.001227$ and $f_{N}^{\text {model }}=0.0006921$; for $(0,4,12) f_{N}=0.3540$ and $f_{N}^{\text {model }}=0.4786$; for $(0,6,12) f_{N}=0.02870$ and $f_{N}^{\text {model }}=$ 0.02805 ; for $(1,6,12) f_{N}=0.1675$ and $f_{N}^{\text {model }}=0.17825$. The differences are significant but typically do not exceed $40 \%$. Better agreements are found for states $(0,6,12)$ and $(1,6,12)$. The discrepancies could be due to the fact that the outer shells are not regular polygons (as assumed in the model) and, in fact, these systems would be modeled more precisely by considering the outer shell as composed of $N_{\text {out }} / N_{\text {in }}$ regular polygons with $N_{\text {in }}$ sides. This argument seems to be supported

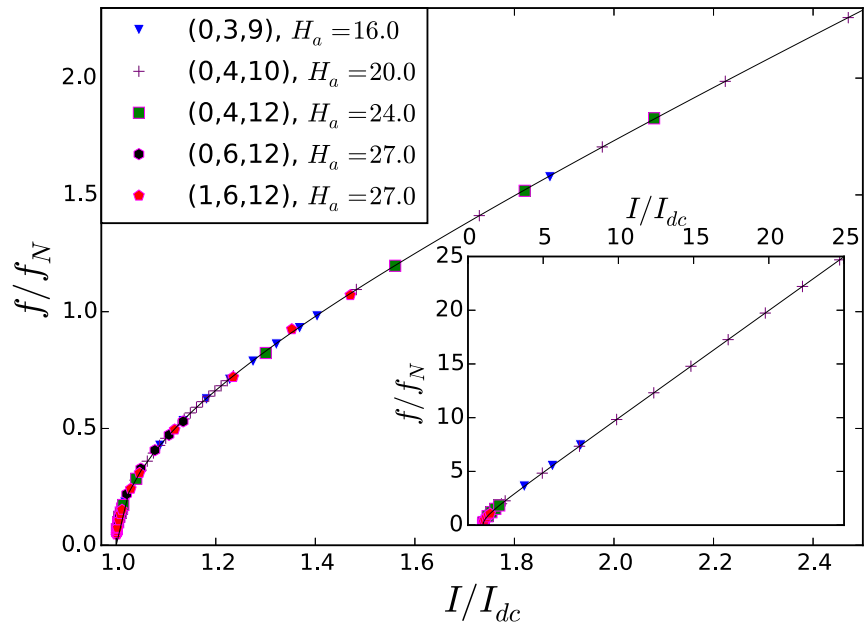

FIG. 14. Frequency of the angular frequency peaks for inner and outer vortex shells as a function of the applied current. The solid line depicts the $\sqrt{\left(I / I_{\mathrm{dc}}\right)^{2}-1}$ dependence. The inset shows the same for a larger range of currents.

by the larger differences observed in the $(0,3,9)$ and $(0,4,12)$ configurations, where the outer shells are, respectively, similar to a rounded triangle and a rounded square [cf. Figs. 9(a) and 9 (b)]. On the other hand, the $(0,6,12)$ and $(1,6,12)$ configurations have nearly circular outer shells [see Figs. 9(c) and 9(d)].

We also studied the angular velocities of the vortices as a function of time obtained from the simulations and compared them to the ones predicted by our model. The agreement is not as good as in the vortex-antivortex case. However, it is still reasonable if we consider the time scaled by $T_{\text {fit }}$ for the simulation results, as well as $t / T_{\text {model }}$ for the angular velocity dependence from the model. An example is shown in Fig. 15 for $(0,4,12)$ configuration at $H_{a}=24$, where the angular velocities $\omega$ of the vortices are depicted as functions of time divided by $T_{\text {fit }}$ for $I=0.01925, I=0.0195, I=0.02$, and $I=0.025$. $\omega$ is divided by $I$ for better visualization. The results from the model, with time divided by $T_{\text {model }}$, are shown by solid lines, where we used $I_{\mathrm{dc}}=0.019225$. Similarly to the vortex-antivortex case, $\omega$ is sharply peaked for currents just

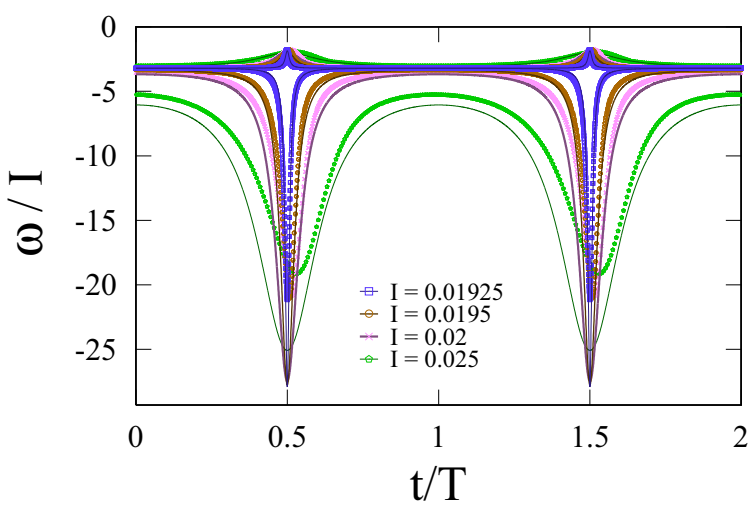

FIG. 15. Angular vortex velocity as function of time for the $(0,4,12)$ configuration at $H_{a}=24$, for different applied currents. Solid lines (points) depicts the results from our model (simulations). Time is divided by the respective period of motion, $T_{\text {model }}$ and $T_{\text {fit }}$, which are related by $T_{\text {fit }} / T_{\text {model }}=f_{N}^{\text {model }} / f_{N}$. 
above $I_{\mathrm{dc}}$ and presents smoother oscillations as $I$ increases. These peaks appear when vortices in one shell overcome the potential well produced by the vortices in the other shell. One of the differences between this situation and the one with vortices and antivortices is the fact that $\omega$ is strictly negative in the former, meaning vortices always rotate in the same direction. The agreement between model and simulation is good for $I$ close to $I_{\mathrm{dc}}$ and worsens as $I$ increases, but maintains a qualitative agreement. The other configurations studied present similar features.

One aspect which is observed in the rotation of shells of vortices-and which is negligible in the configurations with both vortices and antivortices-is that the peaks in $\omega$ for the outer vortices do not appear at the same time. This means that, contrary to our model assumption, here intrashell motion is not negligible. Nonetheless, even without including this effect, we find a qualitative agreement between the simulations and the model.

\section{CONCLUSIONS}

In this paper, we addressed theoretically the problem of the vortex shell dynamics in superconducting disks in the Corbino setup, focusing on configurations comprising only two vortex shells. The cases with the vortex and the antivortex shells (as found under magnetic dots) as well as with only vortices were studied analytically and by numerical simulations. We first developed an analytic model which treats each shell as a RB interacting with the other shell. The model is exact for shells with same numbers of vortices or antivortices (as long as vortex images induced by the boundary condition can be neglected). For the case of commensurate (but not equal) numbers of vortices in the shells we provide an ansatz for the intershell torque, which can describe reasonably well the interactions between vortices in different shells. In the numerical simulations, we subsequently considered the full many-vortex problem, without the rigid-shell constraint.

Our calculations provide evidences for two different phases of the rotating vortex system induced by the nonuniformity of the radial current density in the Corbino geometry: a $\mathrm{RB}$ rotation for $I<I_{\mathrm{dc}}$ and an intershell relative rotation for $I>I_{\mathrm{dc}}$, where $I_{\mathrm{dc}}$ is the critical current separating both phases. In the former, the whole configuration rotates with a prescribed angular velocity $\omega_{\mathrm{RB}}$, whose value is determined by the total vorticity, $L$, and the vortex radial positions. Therefore, if $L=0$ (which is the case for equal number of vortices and antivortices) the configuration does not rotate $\left(\omega_{\mathrm{RB}}=0\right)$ for $I<I_{\mathrm{dc}}$. Above $I_{\mathrm{dc}}$ vortices in the inner shell, which are submitted to stronger stresses, detach from the vortices (or antivortices) in the outer shell, rotate faster, and reposition themselves close to the next vortices (or antivortices) in the outer shell. For the case of configurations containing vortices and antivortices, the attractive vortex-antivortex interaction drags the antivortex shell along with the vortex shell most of the time for currents just above $I_{\mathrm{dc}}$. As the current is further increased, the induced Lorentz force becomes stronger and the dragging effect diminishes until a point where the antivortex shell rotates (on average) in the direction opposite to the vortex shell rotation.
For all the studied configurations (with vortices and antivortices as well as those with only vortices), this process repeats periodically with a frequency proportional to $\sqrt{I^{2}-I_{\mathrm{dc}}^{2}}$. The detailed structure of each shell enters the model through $I_{\mathrm{dc}}$ and the proportionality factor. This result, as well as the time evolution of the vortex angular velocities, was predicted by our two-rigid-shell model and confirmed by the numerical simulations. However, the quantitative agreement between theory and simulation is poorer for the case of configurations containing only vortices. This is, in part, due to significant intrashell displacements within the outer vortex shell which were not considered in our two-shell model. In spite of that, the major features of the shell dynamics remains well described by the model.

Finally, we would like to stress that the predictions of our model can be tested in transport experiments performed on thin disks of low-pinning superconductors in the Corbino geometry. As we have pointed out in the text, the voltage drop between suitably placed point contacts gives a direct measure of the mean angular velocity of each vortex shell separately. For instance, the linear (in RB phase) and the nonlinear (in the decoupled phase) behaviors of the $V(I)$ characteristics predicted by our model are readily experimentally accessible. The sign inversion of the voltage induced by the change of the rotation direction of the outer antivortex shell in the disk-dipole system could also be directly observed. We also note that, by Fourier analyzing the noise spectra of the voltage measured at currents above $I_{\mathrm{dc}}$, the intershell reconnection frequency could be measured, thereby testing our predicted $\sqrt{I^{2}-I_{\mathrm{dc}}^{2}}$ scaling law.

\section{ACKNOWLEDGMENTS}

This work was supported by the Brazilian Science Agencies CAPES, CNPq, and FACEPE under Grants No. APQ-13811.05/12, No. APQ 2017-1.05/12, and No. APQ-0598/1.0508 and by EU-COST Action No. MP1201 and the Research Foundation-Flanders (FWO).

\section{APPENDIX A: TWO-SHELL CONFIGURATIONS WITH COMMENSURATE NUMBER OF VORTICES AND ANTIVORTICES}

For shells with commensurate number of vortices and antivortices, where $N_{+} / N_{-}=1,2,3 \ldots$, with the possibility of one vortex (of vorticity $L_{0}$ ) in the center of the disk, the vortex and the antivortex shells can be divided in $M=\frac{N_{+}}{N_{-}}+1$ groups of vortices. Further, it is reasonable to assume the vortices in a shell form a regular polygon concentric with the disk main axis [62]. Therefore, the system has $N_{-}$-fold rotational symmetry, with vortices and antivortices placed at the vertices of coaxial regular polygons of $N_{-}$sides. Each vortex position and vorticity can be described by $\left(R_{m} \cos \phi_{m, i}, R_{m} \sin \phi_{m, i}\right)$ and $v_{m}=1\left(v_{m}=-1\right.$ for an antivortex $)$, respectively. Here $\phi_{m, i}=\varphi_{m}+2 \pi i / N_{-}, \varphi_{m}$ is an angular arbitrary position for the $m$ th shell, and $m=1,2, \ldots, M$ and $i=1,2, \ldots, N_{-}$. This vortex distribution is depicted in Fig. 16 for the case of a configuration with six (three) vortices (antivortices) in the inner (outer) shell. This configuration has threefold rotational symmetry and two different groups of vortices. $\varphi_{1}, \varphi_{2}$, and $\varphi_{3}$ 


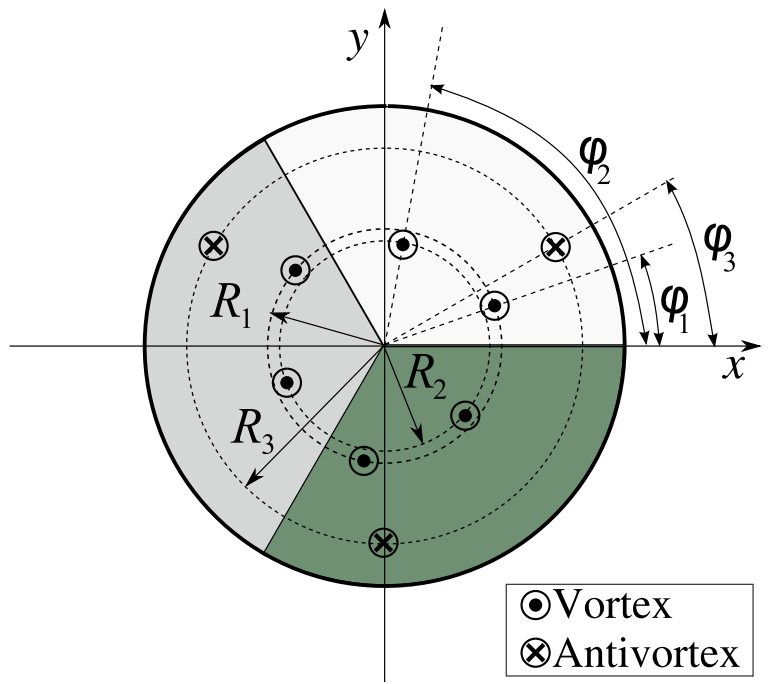

FIG. 16. The two-vortex-shell model for commensurate configurations is represented schematically for the $(0,6,-3)$ vortex state as an example. Circles with dots (crosses) represent vortices (antivortices). Different background colors depict the threefold rotational symmetry of the system. The antivortices are located at radius $R_{3}=R_{-}$and angular positions $\phi_{2}+2 \pi j / 3$ for $j=0,1,2$. The vortices in the inner shell are grouped in $N_{+} / N_{-}=2$ groups at radii $R_{1}$ and $R_{2}$. In each group the angular positions of the vortices are given by $\phi_{s}+2 \pi i / 3$ for $i=0,1,2$ and $s=1,2$.

represent arbitrary angular positions in each shell (which we place at the positions of the given vortex and antivortex).

The following equations of motion describe the dynamics of each vortex ring:

$$
\begin{gathered}
v_{m, r}=-\frac{v_{m} m_{0} R_{m}}{\left(R_{m}^{2}+z_{0}^{2}\right)^{3 / 2}}+\frac{N_{-}-1+2 v_{m} L_{0}}{R_{m}} \\
+\frac{2 N_{-} R_{m}^{2 N_{-}-1}}{1-R_{m}^{2 N_{-}}}+\sum_{\substack{n=1 \\
n \neq m}}^{M} f_{i_{m} n}, \\
R_{m}^{2} \omega_{m}=-v_{m} I+\sum_{\substack{n=1 \\
n \neq m}}^{M} \tau_{i_{m} n},
\end{gathered}
$$

where $v_{m, r}$ is the radial velocity of the vortices in the $m$ th ring, $\varphi_{m n}=\varphi_{m}-\varphi_{n}$,

$$
\begin{aligned}
\tau_{i_{m} n}= & 2 N_{-} v_{m} v_{n} R_{m}^{N_{-}} R_{n}^{N_{-}} \sin N_{-} \varphi_{m n} \\
& \times\left[\frac{1}{D_{N_{-}}\left(R_{m}, R_{n}, \varphi_{m n}\right)}-\frac{1}{D_{N_{-}}\left(1, R_{m} R_{n}, \varphi_{m n}\right)}\right],
\end{aligned}
$$

are the torques produced by the $n$th ring on the $i$ th vortex (or antivortex) in the $m$ th ring, and

$$
\begin{aligned}
f_{i_{m} n}= & 2 N_{-} v_{m} v_{n}\left[\frac{R_{m}^{N_{-}-1}\left(R_{m}^{N_{-}}-R_{n}^{N_{-}} \cos N_{-} \varphi_{m n}\right)}{D_{N_{-}}\left(R_{m}, R_{n}, \varphi_{m n}\right)}\right. \\
& \left.-\frac{R_{m}^{N_{-}-1} R_{n}^{N_{-}}\left(R_{m}^{N_{-}} R_{n}^{N_{-}}-\cos N_{-} \varphi_{m n}\right)}{D_{N_{-}}\left(R_{m} R_{n}, 1, \varphi_{m n}\right)}\right] .
\end{aligned}
$$

is the radial force acting on this vortex. For $N_{-}>1$ the symmetry of the model yields zero force acting on the central vortex, which means this vortex remains static and its equation of motion need not be considered. Moreover, all the vortices within the same ring in the inner shell and all the antivortices in the outer shell move together.

The above equations allow us to find the stable positions of vortices and antivortices in the case of zero applied current ( $I=0$ ) by setting $v_{m}=0$ and $\omega_{m}=0$ for each ring. First, it is reasonable to suppose that the vortices are aligned angularly with the antivortex due their mutual attraction. Therefore, $\varphi_{+}-\varphi_{-}=\varphi=0$ and the equations related to the angular positions are disregarded, leaving one to solve a system of two equations,

$$
\begin{aligned}
& -\frac{v_{m} m_{0} R_{m}^{2}}{\left(R_{m}^{2}+z_{0}^{2}\right)^{3 / 2}}+N_{-}-1+\frac{2 N_{-} R_{m}^{2 N_{-}}}{1-R_{m}^{2 N_{-}}} \\
& \quad+2 N_{-} v_{m} v_{n} R_{m}^{N_{-}}\left[\frac{1}{R_{m}^{N_{-}}-R_{n}^{N_{-}}}-\frac{R_{n}^{N_{-}}}{1-R_{m} R_{n}}\right]=0,
\end{aligned}
$$

for $m=+,-$ and $n=-,+$, respectively.

The radial positions of configurations containing two shells with the same number of vortices and antivortices for $I=$ 0 were found by solving the above system of equations. Specifically, we solved for $L_{0}=0,1$, and $N=2,3,4,5,6$, i.e., the vortex states $(0,2,-2),(0,3,-3),(1,3,-3)$; $(0,4,-4),(1,4,-4) ;(0,5,-5),(1,5,-5) ;(0,6,-6)$; and $(1,6,-6) \cdot m_{0}$ and $z_{0}$ were varied in order to find the regions were these states are stable. Therefore, we find the radial positions of the two vortex shells as a function of $m_{0}$ and $z_{0}$.

\section{APPENDIX B: INTEGRATION OF THE INTERSHELL EQUATION OF MOTION}

In order to solve Eq. (23), one can substitute $\vartheta=2 \arctan \zeta$. Therefore, $\frac{d \vartheta}{d t}=2 \frac{d \zeta}{d t} /\left(1+\zeta^{2}\right)$, sin $\vartheta=2 \zeta /\left(1+\zeta^{2}\right)$, and the equation can be rewritten as

$$
2 \frac{d \zeta}{d t}=K I\left[\left(\zeta-\frac{I_{\mathrm{dc}}}{I}\right)^{2}+c^{2}\right],
$$

where $c^{2}=1-\left(\frac{I_{\mathrm{dc}}}{I}\right)^{2}$. By making $u=\zeta-\frac{I_{\mathrm{dc}}}{I}$ we finally find

$$
\frac{K I t}{2}=\int \frac{d u}{u^{2}+c^{2}}= \begin{cases}-\frac{\operatorname{arctanh}(u / c)}{c}, & \text { for } I<I_{\mathrm{dc}} \\ -u^{-1}, \quad & \text { for } I=I_{\mathrm{dc}} \\ \frac{\arctan (u / c)}{c}, & \text { for } I>I_{\mathrm{dc}}\end{cases}
$$

After rewriting this equation back in terms of $\vartheta=N \varphi$, we arrive at Eqs. (25), (26), and (27).

\section{APPENDIX C: STATIONARY INTERSHELL SEPARATION}

In order to show that Eq. (24) is the limit of Eqs. (25), (26), and (27) for $t \gg T$ and $I<I_{\mathrm{dc}}$, we first notice that $f\left(I<I_{\mathrm{dc}}, t \gg T\right) \approx\left(1-\tilde{I}^{2}\right)^{-1 / 2}$. Therefore,

$$
\begin{aligned}
\zeta(t \gg T) & =\tan \frac{\vartheta_{\text {stat }}}{2} \\
& =\frac{\sqrt{1-\tilde{I}^{2}} \tan \frac{\vartheta_{0}}{2}+\tilde{I}-\tan \frac{\vartheta_{0}}{2}}{\sqrt{1-\tilde{I}^{2}}+1-\tilde{I} \tan \frac{\vartheta_{0}}{2}},
\end{aligned}
$$


where $\vartheta=N \varphi$. If we add and subtract $\tilde{I} /\left(1+\sqrt{1-\tilde{I}^{2}}\right)$ to the RHS of the above equation, we find

$$
\tan \frac{\vartheta_{\text {stat }}}{2}=\frac{\tilde{I}}{1+\sqrt{1-\tilde{I}^{2}}}
$$

Therefore,

$$
\sin \vartheta_{\text {stat }}=\tilde{I},
$$

which results in Eq. (24).

\section{APPENDIX D: MOTION OF SHELLS WITH ARBITRARY RADII FOR $N_{+}=N_{-}$}

The intershell dynamics can be understood for same number of vortices and antivortices by solving Eq. (20). Here we find the solution of Eq. (20) valid for any $R_{+}<R_{-}$.

In the stationary regime we have $\omega_{+}-\omega_{-}=0=\frac{d \vartheta}{d t}$. By using this condition, and Eqs. (21) and (22), we obtain

$$
N \varphi_{\text {stat }}=\arctan \left\{\frac{\sqrt{\left[\left(\frac{I}{I_{\mathrm{dc}}}\right)^{2}+\left(\frac{I}{N}\right)^{2}\right]\left[1-\left(\frac{I}{I_{\mathrm{dc}}}\right)^{2}\right]}-\frac{I}{N}}{1-\left(\frac{I}{I_{\mathrm{dc}}}\right)^{2}-\left(\frac{I}{N}\right)^{2}}\right\},
$$

which is the intershell angular displacement dependence on $I$ for $I<I_{\mathrm{dc}}$ in the stationary regime. The approximated expression given by Eq. (24) holds for $\varrho=R_{+} / R_{-} \ll 1$ or, equivalently, for $I_{\mathrm{dc}} \ll N$.

The time dependence of the intershell angular displacement can be obtained by solving Eq. (20). This equation can be put in the form

$$
K I t-\Delta \vartheta=\int_{\vartheta_{0}}^{\vartheta(t)} \frac{\sin \vartheta d \vartheta}{\frac{I}{I_{\mathrm{dc}} \sin \vartheta_{c}}-\frac{I}{N} \cos \vartheta-\sin \vartheta},
$$

where $\Delta \vartheta=N\left[\varphi(t)-\varphi_{0}\right]$ and $\varphi_{0}=N \varphi(0)$. The integral in the RHS is tabulated [63] and after some algebraic manipulation we arrive at an equation which relates $\varphi$ with time,

$$
\begin{aligned}
(1+ & \left.\frac{I^{2}}{N^{2}}\right) K t \\
= & \frac{\left(\varphi-\varphi_{0}\right) I}{N}+g(\varphi)-g\left(\varphi_{0}\right) \\
& +\ln \left[\frac{N I-I_{\mathrm{dc}} \sin \vartheta_{c}(I \cos N \varphi+N \sin N \varphi)}{N I-I_{\mathrm{dc}} \sin \vartheta_{c}\left(I \cos N \varphi_{0}+N \sin N \varphi_{0}\right)}\right],
\end{aligned}
$$

where

$$
g(\varphi)=\left\{\begin{array}{cl}
\frac{2 \arctan \left[Z(\varphi) / \sin \vartheta_{c} \sqrt{I^{2}-I_{\mathrm{dc}}^{2}}\right]}{\sin \vartheta_{c} \sqrt{I^{2}-I_{\mathrm{dc}}^{2}}}, & \text { for } I>I_{\mathrm{dc}}, \\
\frac{-2 \operatorname{arctanh}\left[Z(\varphi) / \sin \vartheta_{c} \sqrt{I_{\mathrm{dc}}^{2}-I^{2}}\right]}{\sin \vartheta_{c} \sqrt{I_{\mathrm{dc}}^{2}-I^{2}}}, & \text { for } I<I_{\mathrm{dc}}, \\
-\frac{2}{Z(\varphi)}, & \text { for } I=I_{\mathrm{dc}},
\end{array}\right.
$$

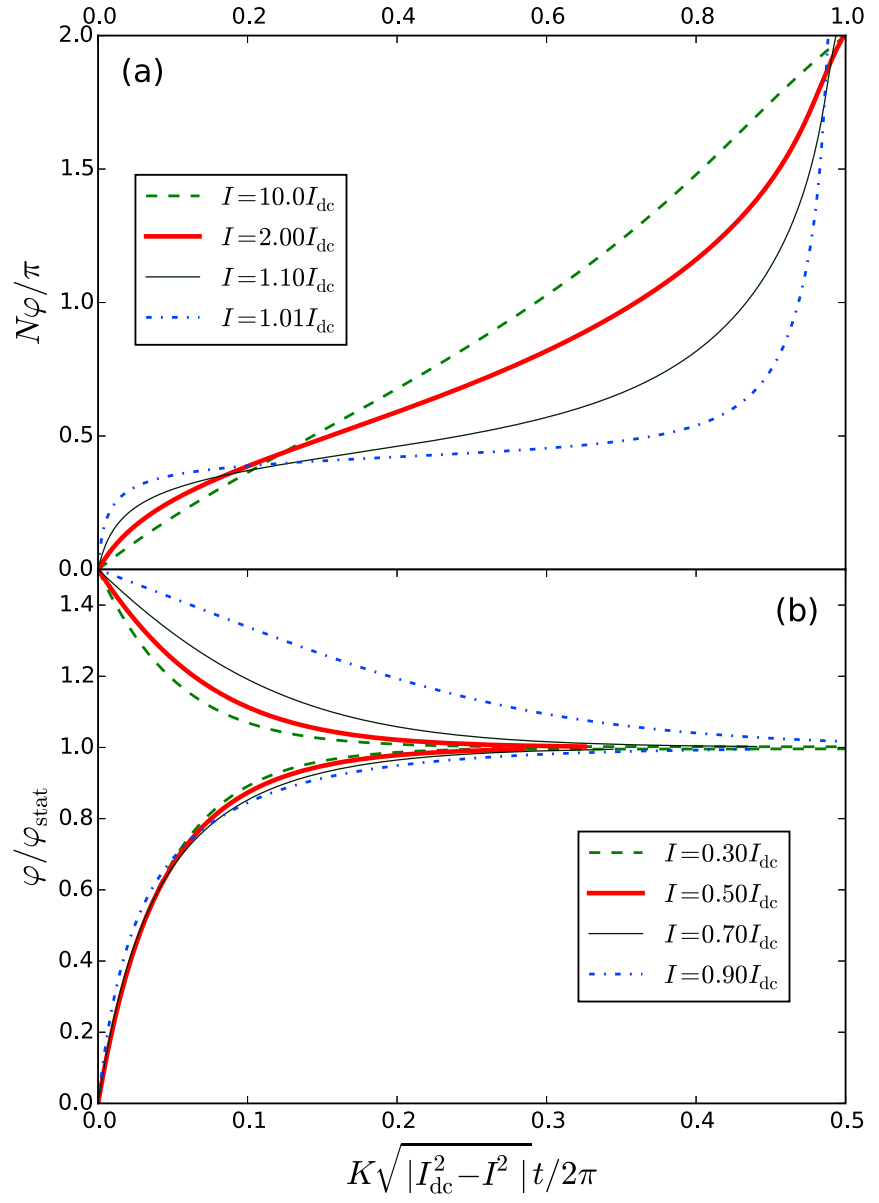

FIG. 17. The intershell angular displacement $\varphi$ as a function of time for several values of the applied current for $N=3$ and $R_{+}=0.1$ and $R_{-}=0.2$. (a) $\varphi(t)$ for $I>I_{\mathrm{dc}}$, depicting the periodic dependence of $\varphi$ on $t$. (b) For $I<I_{\mathrm{dc}}$ the initial intershell displacement converges to the stationary values at times of the order $2 \pi / K \sqrt{I_{\mathrm{dc}}^{2}-I^{2}}$. The behavior of $\varphi$ is similar to the ones shown for $R_{+} \ll R_{-}$, although the oscillatory motion has a small deviation from the expected period $T=2 \pi / K \sqrt{I^{2}-I_{\mathrm{dc}}^{2}}$.

with

$$
Z(\varphi)=\left(1+\frac{I_{\mathrm{dc}}}{N} \sin \vartheta_{c}\right) I \tan \frac{N \varphi}{2}-I_{\mathrm{dc}} \sin \vartheta_{c} .
$$

The time dependence of $\varphi$ is shown in Fig. 17 for $N=3$, and $R_{+}=0.1$ and $R_{-}=0.2$ (these values where chosen with the purpose of presenting the case of arbitrary $\varrho=R_{+} / R_{-}$, in spite of the fact that they are not related to any stable configuration). As in the case for $R_{+} \ll R_{-}$, there are two regimes corresponding to the $\mathrm{RB}$ rotation $\left(I \leqslant I_{\mathrm{dc}}\right)$ and to relative intershell motion $\left(I>I_{\mathrm{dc}}\right)$. In the former [depicted in Fig. 17(b)] any initial value of $\varphi$ will converge to its stationary values [given by Eq. (D1)] as soon as $t$ approaches $2 \pi / K \sqrt{I_{\mathrm{dc}}^{2}-I^{2}}$. On the other hand, for $I>I_{\mathrm{dc}}$ [cf. Fig. 17(a)] $\varphi$ keeps increasing with time, which is a signature of the intershell angular periodic motion. In fact, $\varphi(t)$ behaves similarly to the $R_{+} \ll R_{-}$case (see Sec. III), although with a period slightly different from $T=2 \pi / K \sqrt{I^{2}-I_{\mathrm{dc}}^{2}}$ [see Eq. (28)] calculated for $R_{+} \ll R_{-}$. 
[1] A. E. Koshelev and V. M. Vinokur, Phys. Rev. Lett. 73, 3580 (1994).

[2] U. Yaron, P. L. Gammel, D. A. Huse, R. N. Kleiman, C. S. Oglesby, E. Bucher, B. Batlogg, D. J. Bishop, K. Mortensen, and K. N. Clausen, Nature (London) 376, 753 (1995).

[3] J. Zhang, L. E. De Long, V. Majidi, and R. C. Budhani, Phys. Rev. B 53, R8851 (1996).

[4] S. Ryu, M. Hellerqvist, S. Doniach, A. Kapitulnik, and D. Stroud, Phys. Rev. Lett. 77, 5114 (1996).

[5] H. Fangohr, S. J. Cox, and P. A. J. de Groot, Phys. Rev. B 64, 064505 (2001).

[6] C. Reichhardt and C. J. Olson Reichhardt, Phys. Rev. Lett. 103, 168301 (2009).

[7] G. R. Berdiyorov, M. V. Milošević, and F. M. Peeters, Phys. Rev. B 79, 184506 (2009).

[8] G. R. Berdiyorov, A. D. Hernández-Nieves, M. V. Milošević, F. M. Peeters, and D. Domínguez, Phys. Rev. B 85, 092502 (2012).

[9] G. R. Berdiyorov, M. V. Milošević, M. L. Latimer, Z. L. Xiao, W. K. Kwok, and F. M. Peeters, Phys. Rev. Lett. 109, 057004 (2012).

[10] B. N. J. Persson, Phys. Rev. B 48, 18140 (1993).

[11] D. Nakamura, T. Kubo, S. Kitamura, and A. Maeda, J. Phys.: Condens. Matter 22, 445702 (2010).

[12] A. Maeda, Y. Inoue, H. Kitano, S. Savel'ev, S. Okayasu, I. Tsukada, and F. Nori, Phys. Rev. Lett. 94, 077001 (2005).

[13] L. Corte, P. M. Chaikin, J. P. Gollub, and D. J. Pine, Nat Phys 4, 420 (2008).

[14] S. Okuma, Y. Tsugawa, and A. Motohashi, Phys. Rev. B 83, 012503 (2011).

[15] Y. Paltiel, E. Zeldov, Y. Myasoedov, M. L. Rappaport, G. Jung, S. Bhattacharya, M. J. Higgins, Z. L. Xiao, E. Y. Andrei, P. L. Gammel, and D. J. Bishop, Phys. Rev. Lett. 85, 3712 (2000).

[16] S. F. W. R. Rycroft, R. A. Doyle, D. T. Fuchs, E. Zeldov, R. J. Drost, P. H. Kes, T. Tamegai, S. Ooi, and D. T. Foord, Phys. Rev. B 60, R757 (1999).

[17] Y. Paltiel, E. Zeldov, Y. N. Myasoedov, H. Shtrikman, S. Bhattacharya, M. J. Higgins, Z. L. Xiao, E. Y. Andrei, P. L. Gammel, and D. J. Bishop, Nature (London) 403, 398 (2000).

[18] J. Mirković, S. E. Savel'ev, E. Sugahara, and K. Kadowaki, Phys. Rev. Lett. 86, 886 (2001).

[19] S. Okuma, K. Kashiro, Y. Suzuki, and N. Kokubo, Phys. Rev. B 77, 212505 (2008).

[20] S. Okuma, S. Morishima, and M. Kamada, Phys. Rev. B 76, 224521 (2007).

[21] M. C. Marchetti, Phys. C (Amsterdam, Neth.) 341-348, 991 (2000).

[22] G. Crabtree, D. Lopez, W. Kwok, H. Safar, and L. Paulius, J. Low Temp. Phys. 117, 1313 (1999).

[23] P. Benetatos and M. C. Marchetti, Phys. Rev. B 65, 134517 (2002).

[24] D. López, W. K. Kwok, H. Safar, R. J. Olsson, A. M. Petrean, L. Paulius, and G. W. Crabtree, Phys. Rev. Lett. 82, 1277 (1999).

[25] G. W. Crabtree, Nat. Mater. 2, 435 (2003).

[26] M.-C. Miguel and S. Zapperi, Nat. Mater. 2, 477 (2003).

[27] A. Furukawa and Y. Nisikawa, Phys. Rev. B 73, 064511 (2006).

[28] S. Okuma, Y. Yamazaki, and N. Kokubo, Phys. Rev. B 80, 220501(R) (2009).

[29] M.-Carmen Miguel, A. Mughal, and S. Zapperi, Phys. Rev. Lett. 106, 245501 (2011).
[30] M. Marchetti and D. R. Nelson, Phys. C (Amsterdam, Neth.) 330, 105 (2000).

[31] I. Buttinoni, Z. A. Zell, T. M. Squires, and L. Isa, Soft Matter 11, 8313 (2015).

[32] C. Bairnsfather, C. J. Olson Reichhardt, and C. Reichhardt, Europhys. Lett. 94, 18001 (2011).

[33] A. Libál, B. M. Csíki, C. J. Olson Reichhardt, and C. Reichhardt, Phys. Rev. E 87, 022308 (2013).

[34] C. Reichhardt and C. J. Olson Reichhardt, Phys. Rev. B 84, 174208 (2011).

[35] C. Reichhardt, C. Bairnsfather, and C. J. Olson Reichhardt, Phys. Rev. E 83, 061404 (2011).

[36] A. I. Buzdin and J. P. Brison, Phys. Lett. A 196, 267 (1994).

[37] P. A. Venegas and E. Sardella, Phys. Rev. B 58, 5789 (1998).

[38] L. R. E. Cabral, B. J. Baelus, and F. M. Peeters, Phys. Rev. B 70, 144523 (2004).

[39] I. V. Grigorieva, W. Escoffier, J. Richardson, L. Y. Vinnikov, S. Dubonos, and V. Oboznov, Phys. Rev. Lett. 96, 077005 (2006).

[40] M. R. Connolly, M. V. Milošević, S. J. Bending, J. R. Clem, and T. Tamegai, Europhys. Lett. 85, 17008 (2009).

[41] V. R. Misko and F. M. Peeters, Phys. Rev. B 74, 174507 (2006).

[42] N. S. Lin, V. R. Misko, and F. M. Peeters, Phys. Rev. Lett. 102, 197003 (2009).

[43] N. S. Lin, V. R. Misko, and F. M. Peeters, Phys. Rev. B 81, 134504 (2010).

[44] D. S. Golubović, W. V. Pogosov, M. Morelle, and V. V. Moshchalkov, Europhys. Lett. 65, 546 (2004).

[45] C. Carballeira, V. V. Moshchalkov, L. F. Chibotaru, and A. Ceulemans, Phys. Rev. Lett. 95, 237003 (2005).

[46] D. S. Golubović, M. V. Milošević, F. M. Peeters, and V. V. Moshchalkov, Phys. Rev. B 71, 180502 (2005).

[47] J. S. Neal, M. V. Milošević, S. J. Bending, A. Potenza, L. San Emeterio, and C. H. Marrows, Phys. Rev. Lett. 99, 127001 (2007).

[48] M. V. Milošević, S. V. Yampolskii, and F. M. Peeters, Phys. Rev. B 66, 024515 (2002).

[49] M. V. Milošević and F. M. Peeters, Phys. Rev. B 68, 024509 (2003).

[50] M. V. Milošević, G. R. Berdiyorov, and F. M. Peeters, Phys. Rev. B 75, 052502 (2007).

[51] A. Y. Aladyshkin, A. V. Silhanek, W. Gillijns, and V. V. Moshchalkov, Supercond. Sci. Technol. 22, 053001 (2009).

[52] B. R. de Aquino, L. R. E. Cabral, C. C. de Souza Silva, J. Albino Aguiar, M. V. Milošević, and F. M. Peeters, Phys. C (Amsterdam, Neth.) 479, 115 (2012).

[53] V. A. Schweigert, F. M. Peeters, and P. S. Deo, Phys. Rev. Lett. 81, 2783 (1998).

[54] A. Kanda, B. J. Baelus, F. M. Peeters, K. Kadowaki, and Y. Ootuka, Phys. Rev. Lett. 93, 257002 (2004).

[55] B. Xu, M. V. Milošević, S.-H. Lin, F. M. Peeters, and B. Jankó, Phys. Rev. Lett. 107, 057002 (2011).

[56] J. Bardeen and M. J. Stephen, Phys. Rev. 140, A1197 (1965).

[57] In fact, we compared this simple assumption for the intershell torque with the exact torque calculated from simulation data. We found good agreement for those configurations with smaller deviations from a regular polygon and axisymmetric with the disk axis, with $\tau_{\max }$ as the only fitting parameter. However, we could not derive $\tau_{\max }$, and, therefore, $I_{\mathrm{dc}}$, from direct calculations based only on the static configuration $(I=0)$. The reason 
stems from the fact that the vortex relative positions (i.e., the configuration deforms) as $I$ varies.

[58] J. R. Clem, Phys. Rep. 75, 1 (1981).

[59] If there were the same number of vortices and antivortices in different shells, we would find zero voltage for $I<I_{\mathrm{dc}}$, since $\omega_{\mathrm{RB}}=0$ in this case.

[60] L. R. E. Cabral and J. A. Aguiar, Phys. Rev. B 80, 214533 (2009).

[61] B. J. Baelus, L. R. E. Cabral, and F. M. Peeters, Phys. Rev. B 69, 064506 (2004).
[62] However, while the assumptions of zero radial position of the central vortex and coaxial vortex shells are reasonable for $N_{-}>$ 1 , where $N_{-}$is the number of antivortices in the outer shell, this is not necessarily the case for $N_{-}=1$. In this latter case the antivortex distorts the vortex shell more strongly than in the $N_{-}>1$ cases, which may dislocate the central vortex from the disk center, breaking the rotational symmetry.

[63] I. S. Gradshteyn and I. M. Ryzhik, Table of Integrals, Series, and Products, 4th ed. (Academic Press, San Diego, 1965). 